\title{
Implicit analytic solutions for a nonlinear fractional partial differential beam equation
}

\author{
Konstantinos B. Liaskos*, Athanasios A. Pantelous† Ioannis A. Kougioumtzoglou‡ \\ Antonios T. Meimaris , Antonina Pirrotta
}

\begin{abstract}
In this paper, we derive implicit analytic solutions for a class of nonlinear fractional partial differential equation (PDE) which models the dynamics of deterministically excited viscoelastic nonlinear Euler-Bernoulli beams. Precisely, the initial-boundary value problem (IBVP) for the corresponding $\mathrm{PDE}$ is reduced to an initial value problem for a nonlinear ordinary differential equation in a Hilbert space. Then, by employing the cosine and sine families of operators, a variation of parameters representation of the solution map is introduced. Due to the presence of a nonlinear term, a local fixed point theorem is employed to prove the local existence and uniqueness of the solution. Relying on the regularity properties of cosine and sine families, taking into account the form of the nonlinear term, and considering the properties of the fractional derivative, the solution map of the abstract problem is cast into a derivative-free implicit analytic solution for the IBVP. Results corresponding to the limiting purely elastic and purely viscous cases are also provided.
\end{abstract}

*Department of Mathematics, College of Engineering and Technology, American University of the Middle East, Kuwait, e-mail: Konstantinos.Liaskos@aum.edu.kw

${ }^{\dagger}$ Corresponding author: Department of Econometrics and Business Statistics, Monash Business School, Monash University, Wellington Rd, Clayton, Victoria 3800, Australia, e-mail: Athanasios.Pantelous@monash.edu

${ }^{\ddagger}$ Department of Civil Engineering and Engineering Mechanics, The Fu Foundation School of Engineering and Applied Science, Columbia University, 500 West 120th Street, New York, NY 10027, USA, e-mail: ikougioum@columbia.edu

${ }^{\S}$ Department of Econometrics and Business Statistics, Monash Business School, Monash University, Wellington Rd, Clayton, Victoria 3800, Australia, e-mail: Antonios.Meimaris@monash.edu

IDipartimento di Ingegneria (DI), Università degli Studi di Palermo, Viale delle Scienze, Palermo 90128, Italy and Department of Mathematical Sciences, University of Liverpool, Liverpool L697ZL, UK, e-mails: Antonina.Pirrotta@unipa.it, Pirrotta@liverpool.ac.uk 
Keywords: Nonlinear beam; Fractional derivative; Partial differential equation; Cosine and sine families of operators; Implicit analytic solution

\section{Introduction}

Since the beginning of 20th century (e.g. [29, 19]), fractional calculus has been developed and applied to several real-world problems in many important areas of science and engineering. These include, but are not limited to, the mechanical properties modeling of viscoelastic materials, the frequencydependent acoustic wave propagation in porous media, the performance enhancement of nano-image processing, as well as the response analysis of various diverse mechanical systems endowed with fractional derivative; see for instance, [26, 1, 28, 34, 11, 21, 12, 37, 18].

Specifically, the beam bending problem has received considerable attention in the field of engineering mechanics over the past few decades (e.g. [33, 20, 35, 2, 7]). This is primarily due to both its importance as a versatile structural model, and its use as a benchmark problem with closed-form solutions for assessing the performance of numerical solution schemes. Nevertheless, only recently there have been efforts towards determining the deterministic and/or stochastic response of bending beams endowed with fractional derivative elements modeling (e.g. [10, 36, 13, 31, 32, 8]).

In this regard, the authors derived recently implicit analytic solutions for a stochastically excited linear beam with fractional derivative terms [25]. In this paper, the solution methodology developed in [25] is generalized to account for nonlinearities present in the beam modeling. From a mathematical perspective, implicit analytic solutions are derived for the nonlinear fractional partial differential equation (PDE) modeling the dynamics of a deterministically excited beam. In particular, following [36], the equation governing the relatively large deflection $v(t, x)$ of a deterministically excited beam of length $L$, and subjected also to a nonlinear axial force $N$, takes the form

$$
\begin{aligned}
\rho A \frac{\partial^{2} v}{\partial t^{2}}(t, x)+c_{0} \partial_{t}^{\alpha} v(t, x)+E I \frac{\partial^{4} v}{\partial x^{4}}(t, x)-N \frac{\partial^{2} v}{\partial x^{2}}(t, x) & =q(t, x), \text { a.e. on }(0, T) \times(0, L), \\
v(0, x)=v_{0}(x), \frac{\partial v}{\partial t}(0, x) & =v_{1}(x), \text { a.e. on }(0, L), \\
v(t, 0)=v(t, L)=\frac{\partial^{2} v}{\partial x^{2}}(t, 0)=\frac{\partial^{2} v}{\partial x^{2}}(t, L) & =0, \text { a.e. on }(0, T) .
\end{aligned}
$$


where by ${ }_{0} \partial_{t}^{\alpha} v(t, x)$ we denote the Caputo fractional partial derivative $[5,6]$

$$
{ }_{0} \partial_{t}^{\alpha} v(t, x)=\frac{1}{\Gamma(1-\alpha)} \int_{0}^{t} \frac{\frac{\partial v}{\partial s}(s, x)}{(t-s)^{\alpha}} d s, \quad 0<\alpha<1 .
$$

Note that Eq. (1) contains the appropriate initial conditions (i.e., initial deflection and slope along the beam length), while the boundary conditions correspond to a simply supported beam [33, 25]. Further, the fractional derivative describes the viscoelastic material behavior [29, 10, 31, 32], with the limiting cases $\alpha=0$ and $\alpha=1$ representing a purely elastic and a purely viscous behavior, respectively; and $c_{0}$ is a parameter of the fractional derivative term. In the initial-boundary value problem (IBVP) (1), the elastic modulus, the moment of inertia of the cross section, the mass density, the cross-sectional area, and the nonlinear axial force term are denoted by $E, I \rho, A$ and $N$, respectively, i.e.,

$$
N=\frac{E A}{2 L} \int_{0}^{L}\left|\frac{\partial v}{\partial x}(t, x)\right|^{2} d x
$$

which is associated with the assumption of relatively large deflection. Furthermore, the excitation $q(t, x)$ is considered as a square integrable function on $x$ and bounded on $t$. System (1) is an IBVP for a fractional PDE. Considering that we can replace the integrals and derivatives (with respect to the spatial variables) by operators in a proper functional space motivates the abstract treatment of the IBVP (1). Further, considering the Hilbert space, $L^{2}(0, L)$, problem (1) is written as an initial value problem (IVP) of a second-order nonlinear ordinary differential equation (ODE) in $L^{2}(0, L)$. In our approach, the abstract theory of second-order equations is employed instead of reducing the problem further to a first-order one. This is possible due to the fact that the governing operator of the system (i.e., the fourth-order differential operator) and the corresponding cosine and sine families can be explicitly evaluated, as well as their actions on its coefficients. Next, exploiting the abstract theory and representing the nonlinear term and the fractional term in suitable forms, a variation of parameters representation is introduced for obtaining the solution map of the IBVP (1). The derived methodology and results can be considered as an important extension of [17] and [27] (see also [9, 3] for additional relevant references) to account for fractional derivative terms, and as an interesting generalization of the authors' previous work [25] to account for nonlinearities in the fractional PDE.

The paper is organized as follows: The main results of the paper are presented in Section 2. In particular, in Section 2.1 the viscous case is considered, where the abstract form of the equation is derived and the nonlinear term is accounted for. In Section 2.2, attention is directed to the general viscoelastic case and the treatment of the fractional derivative term. The elastic case is presented in Section 2.3 under two distinct perspectives. In all three cases, a variation of parameters representation 
for the solution map of the problem is obtained and a local fixed point theorem is applied. The time regularity of the solutions is considered separately in Section 3. Concluding remarks are presented in Section 4 .

\section{Main results}

For the reduction of an IBVP of a PDE (or a system of PDEs) to an IVP for an ODE in a suitable Hilbert (or Banach) space, the interesting reader is directed to $[14,30,23,24]$ and references therein. Regarding fundamental concepts on fractional derivative representations, as well as the utilization of the Caputo fractional derivative in engineering applications, a detailed presentation can be found in $[10,36,25]$ and references therein. A thorough review of the abstract second order Cauchy problem in Banach spaces and the properties of the associated cosine and sine families of operators can be found in $[15,16,38,39,4]$. In what follows, we make use of Theorem 2.2 in [22] for the local existence and uniqueness of a fixed point of the solution map.

To enhance the pedagogical merit of the derived results, we treat first the purely viscous case, then we proceed with the viscoelastic case which is the most general one, and finally, we conclude with the purely elastic case.

\subsection{Purely viscous case}

In the case of classical viscous damping $(\alpha=1)$, the IBVP (1) becomes:

$$
\left.\begin{array}{c}
\rho A \frac{\partial^{2} v}{\partial t^{2}}(t, x)+c \frac{\partial v}{\partial t}(t, x)+E I \frac{\partial^{4} v}{\partial x^{4}}(t, x)-N \frac{\partial^{2} v}{\partial x^{2}}(t, x) \quad=q(t, x), \text { a.e. on }(0, T) \times(0, L), \\
v(0, x)=v_{0}(x), \frac{\partial v}{\partial t}(0, x)=v_{1}(x), \text { a.e. on }(0, L), \\
v(t, 0)=v(t, L)=\frac{\partial^{2} v}{\partial x^{2}}(t, 0)=\frac{\partial^{2} v}{\partial x^{2}}(t, L) \quad=0, \text { a.e. on }(0, T) .
\end{array}\right\}
$$

To recast equation (2) into an abstract ODE problem in a proper Hilbert space, the standard Hilbert space of all square integrable functions $H=L^{2}(0, L)$ is considered, and the solution map $v:[0, T] \times$ $[0, L] \rightarrow \mathbb{R}$ as $v:[0, T] \rightarrow H$ is identified, where

$$
v(t)(x)=v(t, x), \quad t \in[0, T], x \in[0, L] .
$$


Next, we define the operator $\mathcal{A}: D(\mathcal{A}) \rightarrow H$ as

$$
\mathcal{A} v(t)=\frac{E I}{\rho A} \frac{\partial^{4} v}{\partial x^{4}}(t, \cdot), t \in[0, T]
$$

with

$$
D(\mathcal{A})=\left\{y \in H^{4}(0, L): y(0)=y(L)=y^{\prime \prime}(0)=y^{\prime \prime}(L)=0\right\}
$$

From [17], [27], the self-adjoint operator $\mathcal{A}$ us positive on $H$ with eigenvalues

$$
\lambda_{n}=\frac{E I}{\rho A}\left(\frac{n \pi}{L}\right)^{4}, n \in \mathbb{N}
$$

and corresponding eigenvectors

$$
e_{n}(x)=\sqrt{\frac{2}{L}} \sin \left(\frac{n \pi}{L} x\right), n \in \mathbb{N}, x \in(0, L),
$$

which determine an orthonormal basis of $H=L^{2}(0, L)$. Hence, the operator $\mathcal{A}$ has the spectral representation

$$
\mathcal{A} y=\sum_{n=1}^{\infty} \lambda_{n}<y, e_{n}>_{H} e_{n}, \text { for } y \in D(\mathcal{A})
$$

where

$$
<y, e_{n}>_{H}=\sqrt{\frac{2}{L}} \int_{0}^{L} y(x) \sin \left(\frac{n \pi}{L} x\right) d x
$$

is the inner product in $H=L^{2}(0, L)$. Further, $-\mathcal{A}$ generates a strongly continuous cosine family of operators $C(t), t \in \mathbb{R}$, on $H$, which takes the form

$$
C(t) y=\sum_{n=1}^{\infty} \cos \left(\sqrt{\frac{E I}{\rho A}} \frac{n^{2} \pi^{2}}{L^{2}} t\right)<y, e_{n}>_{H} e_{n}, \text { for } y \in H
$$

Thus, the corresponding strongly continuous sine family of operators $S(t), t \in \mathbb{R}$, on $H$, is of the form

$$
S(t) y=\int_{0}^{t} C(s) y d s=\sum_{n=1}^{\infty} \sqrt{\frac{\rho A}{E I}} \frac{L^{2}}{n^{2} \pi^{2}} \sin \left(\sqrt{\frac{E I}{\rho A}} \frac{n^{2} \pi^{2}}{L^{2}} t\right)<y, e_{n}>_{H} e_{n}, \text { for } y \in H .
$$

For every $t \in \mathbb{R}$, it yields that

$$
\|C(t) y\|_{H}^{2} \leq \sum_{n=1}^{\infty}\left|\cos \left(\sqrt{\frac{E I}{\rho A}} \frac{n^{2} \pi^{2}}{L^{2}} t\right)\right|^{2}\left|<y, e_{n}>\right|_{H}^{2} \leq \sum_{n=1}^{\infty}\left|<y, e_{n}>\right|_{H}^{2}=\|y\|_{H}^{2}, \text { for } y \in H .
$$

Therefore, $\|C(t)\|_{\mathcal{L}_{H}} \leq 1$, for $t \in \mathbb{R}$. Similarly, $M_{S}>0$ can be found such that $\|S(t)\|_{\mathcal{L}(H)} \leq M_{S}$, for $t \in \mathbb{R}$. Hence, for $M=\max \left\{1, M_{S}\right\}$ and $\omega=0$, Proposition 1 in [25] (see also [38]) is satisfied. By utilizing fractional powers of $\mathcal{A}$ (see [17]), which are also self-adjoint operators, the spatial partial derivatives are expressed as

$$
\mathcal{A}^{1 / 2} v(t)=-\sqrt{\frac{E I}{\rho A}} \frac{\partial^{2} v}{\partial x^{2}}(t, \cdot)
$$


with

$$
D\left(\mathcal{A}^{1 / 2}\right)=\left\{y \in H^{2}(0, L): y(0)=y(L)=0\right\}
$$

which has the spectral representation

$$
\mathcal{A}^{1 / 2} y=\sum_{n=1}^{\infty}\left(\lambda_{n}\right)^{1 / 2}<y, e_{n}>_{H} e_{n}, \text { for } y \in D\left(\mathcal{A}^{1 / 2}\right),
$$

and

$$
\mathcal{A}^{1 / 4} v(t)=i \sqrt[4]{\frac{E I}{\rho A}} \frac{\partial v}{\partial x}(t, \cdot)
$$

with

$$
D\left(\mathcal{A}^{1 / 4}\right)=\left\{y \in H^{1}(0, L): y(0)=y(L)=0\right\} .
$$

Since the first order derivative operator, $\frac{\partial v}{\partial x}: D\left(\mathcal{A}^{1 / 4}\right) \rightarrow H$, is skew-adjoint under the assumption that $v(t, \cdot) \in D\left(\mathcal{A}^{1 / 2}\right)$, it follows that

$$
\int_{0}^{L}\left|\frac{\partial v}{\partial x}(t, \cdot)\right|^{2} d x=<\frac{\partial v}{\partial x}(t, \cdot), \frac{\partial v}{\partial x}(t, \cdot)>_{H}=<-\frac{\partial^{2} v}{\partial x^{2}}(t, \cdot), v(t, \cdot)>_{H}=\sqrt{\frac{\rho A}{E I}}<\mathcal{A}^{1 / 2} v(t), v(t)>_{H},
$$

and consequently, for the nonlinear term of the axial force we obtain the important expression

$$
-\frac{N}{\rho A} \frac{\partial^{2} v}{\partial x^{2}}(t, x)=\frac{A}{2 L I}<\mathcal{A}^{1 / 2} v(t), v(t)>_{H} \mathcal{A}^{1 / 2} v(t) .
$$

Setting

$$
\mathcal{B}=\frac{c}{\rho A} \mathcal{I},
$$

where $\mathcal{I}$ is the identity operator on $H$ and considering that $q(t, \cdot) \in H$, the IBVP (2) is now a second order nonlinear $O D E$ in $H$ :

$$
\left.\begin{array}{c}
v^{\prime \prime}(t)+\mathcal{B} v^{\prime}(t)+\mathcal{A} v(t)+\frac{A}{2 L I}<\mathcal{A}^{1 / 2} v(t), v(t)>_{H} \mathcal{A}^{1 / 2} v(t) \quad=\frac{1}{\rho A} q(t), \text { a.e. on }(0, T) \\
v(0)=v_{0}, v^{\prime}(0)=v_{1} .
\end{array}\right\}
$$

At this point, we refer to a result provided in [25] on the commutation of the operator, $\mathcal{A}^{1 / 2}$, with $C(t), S(t), t \in \mathbb{R}$, generated by $-\mathcal{A}$, as well as the uniform boundedness of $\mathcal{A}^{1 / 2} S(t), t \in[0, T]$ (see conditions in $[15,38,25])$.

Lemma 1. [25] Let $y \in D\left(\mathcal{A}^{1 / 2}\right)$. Then, for every $t \in \mathbb{R}$, we have that $C(t) y \in D\left(\mathcal{A}^{1 / 2}\right), S(t) y \in D(\mathcal{A})$ and $C(t) \mathcal{A}^{1 / 2} y=\mathcal{A}^{1 / 2} C(t) y, S(t) \mathcal{A}^{1 / 2} y=\mathcal{A}^{1 / 2} S(t) y, \mathcal{A} S(t) y=\mathcal{A}^{1 / 2} S(t) \mathcal{A}^{1 / 2} y$. Furthermore, the family of bounded operators $\mathcal{A}^{1 / 2} S(t), t \in \mathbb{R}$, on $H$, satisfies that $\left\|\mathcal{A}^{1 / 2} S(t)\right\|_{\mathcal{L}(H)} \leq 1$. 
To define properly the notion of a mild solution for the IVP (21) (when a variation of parameters representation exists), note that the second order spatial derivative operator $\mathcal{A}^{1 / 2}$ is involved in the nonlinear term. Even though the operator $\mathcal{A}$ will not appear in the solution form (since its action is represented by the cosine and sine families), the nonlinear term will appear explicitly in the solution, and in fact, will be convoluted with the sine family. Thus, the mild solution for the IVP (21) should belong (at least) to $D\left(\mathcal{A}^{1 / 2}\right)$. However, taking into account the regularity properties of the sine and cosine families, and under sufficient conditions for the coefficients, $v_{0}, v_{1}, q$, it is also possible to obtain a solution which belongs to $D(\mathcal{A})$. In this regard, note that the square root of the generator $-\mathcal{A}$ is the operator $B=i \mathcal{A}^{1 / 2}$. Considering that $D\left(\mathcal{A}^{1 / 2}\right)=D(B) \subseteq \mathcal{E}$ leads to the result that if $v(t) \in D(\mathcal{A}), t \in[0, T]$, then the term $<\mathcal{A}^{1 / 2} v(t), v(t)>_{H} \mathcal{A}^{1 / 2} v(t) \in D\left(\mathcal{A}^{1 / 2}\right)$, for $t \in[0, T]$. Hence, considering Proposition 1 in [25], the term $S(t)<\mathcal{A}^{1 / 2} v(t), v(t)>_{H} \mathcal{A}^{1 / 2} v(t) \in D(\mathcal{A})$, for $t \in[0, T]$. This type of solution, under the condition that it is also twice differentiable on $(0, T)$, will be defined as a classical solution for the IVP (21) in the Hilbert space $H$. The time regularity of this type of solutions will be treated separately in Section 3.

Definition 1. A twice differentiable function, $v:[0, T] \rightarrow H$, is called a solution of the IVP (21) if

(i) $v(t) \in D(\mathcal{A}), \quad t \in[0, T]$,

(ii) $v(t)=(C(t)+S(t) \mathcal{B}) v_{0}+S(t) v_{1}-\int_{0}^{t} C(t-s) \mathcal{B} v(s) d s$

$$
-\frac{A}{2 L I} \int_{0}^{t} S(t-s)<\mathcal{A}^{1 / 2} v(s), v(s)>_{H} \mathcal{A}^{1 / 2} v(s) d s+\frac{1}{\rho A} \int_{0}^{t} S(t-s) q(s) d s, t \in[0, T] .
$$

Since the solution map in Definition 1 is nonlinear, an appropriate fixed point theorem in a proper Banach space is required in the ensuing analysis. Thus, consider the graph norm space

$$
H_{\mathcal{A}}=\left(D(\mathcal{A}),\|\cdot\|_{\mathcal{A}}\right)
$$

with

$$
\|y\|_{H_{\mathcal{A}}}^{2}=\|y\|_{H}^{2}+\|\mathcal{A} y\|_{H}^{2}, \quad y \in D(\mathcal{A}),
$$

and the Banach space $\mathcal{C}=\left(\mathcal{C}\left(0, T ; H_{\mathcal{A}}\right),\|\|_{\mathcal{C}}\right)$, of all $H_{\mathcal{A}}$-valued, continuous functions equipped with the norm

$$
\|f\|_{\mathcal{C}}=\sup _{t \in[0, T]}\|f(t)\|_{H_{\mathcal{A}}}
$$

For the coefficients $v_{0}, v_{1}, q$, the following assumptions are considered, i.e.,

$$
v_{0} \in D(\mathcal{A}), \quad v_{1} \in D\left(\mathcal{A}^{1 / 2}\right)
$$


and

$$
q \in L^{1}\left(0, T ; H_{\mathcal{A}^{1 / 2}}\right)
$$

Lemma 2. Let $v \in \mathcal{C}$. Then, there is a positive number $M_{\mathcal{A}}<\infty$ such that

$$
\begin{aligned}
& \text { 1. } \sup _{t \in[0, T]}\|v(t)\|_{H} \leq M_{\mathcal{A}} \sup _{t \in[0, T]}\|\mathcal{A} v(t)\|_{H} . \\
& \text { 2. } \sup _{t \in[0, T]}\left\|\mathcal{A}^{1 / 4} v(t)\right\|_{H} \leq M_{\mathcal{A}} \sup _{t \in[0, T]}\|\mathcal{A} v(t)\|_{H} .
\end{aligned}
$$

Proof. Taking into account that

$$
\|v(t)\|_{H}^{2}=\sum_{n=1}^{\infty}\left|<v(t), e_{n}>_{H}\right|^{2}
$$

and considering the spectral representation of $\mathcal{A}^{1 / 4}$, and Eqs. (16) and (17), leads to

$$
\left\|\mathcal{A}^{1 / 4} v(t)\right\|_{H}^{2}=\sqrt{\frac{E I}{\rho A}} \frac{\pi^{2}}{L^{2}} \sum_{n=1}^{\infty} n^{2}\left|<v(t), e_{n}>_{H}\right|^{2} .
$$

Since $v(t) \in D(\mathcal{A}), t \in[0, T]$, by the spectral representation of $\mathcal{A}$, Eqs. (4) and (5), it yields that

$$
\|\mathcal{A} v(t)\|_{H}^{2}=\left(\frac{E I}{\rho A}\right)^{2} \frac{\pi^{8}}{L^{8}} \sum_{n=1}^{\infty} n^{8}\left|<v(t), e_{n}>_{H}\right|^{2} .
$$

Setting $M_{\mathcal{A}}=\max \left\{\left(\frac{E I}{\rho A} \frac{\pi}{L}\right)^{-3 / 4},\left(\frac{E I}{\rho A} \frac{\pi^{4}}{L^{4}}\right)^{-1}\right\}$, the results of Lemma 2 are obtained.

Theorem 1. Suppose that assumptions, (26) and (27) hold, and that the coefficients $v_{0}, v_{1}, q$, satisfy the relations

$$
\begin{gathered}
T \sqrt{1+M_{\mathcal{A}}^{2}}\left[\frac{c}{\rho A}+\frac{3 A}{2 L I} M_{\mathcal{A}}^{2}\left(R+v_{0}^{\star}\right)^{2}\right]<1 \\
\sqrt{1+M_{\mathcal{A}}^{2}}\left[\left(2+\frac{c\left(M_{S}+T\right)}{\rho A}\right) v_{0}^{\star}+v_{1}^{\star}+\frac{T A}{2 L I} M_{\mathcal{A}}^{2}\left(v_{0}^{\star}\right)^{3}+\frac{T}{\rho A} q^{\star}+R T\left(\frac{c}{\rho A}+\frac{3 A}{2 L I} M_{\mathcal{A}}^{2}\left(R+v_{0}^{\star}\right)^{2}\right)\right]<R
\end{gathered}
$$

for some $R>0$ and $T>0$, with $v_{0}^{\star}=\left\|\mathcal{A} v_{0}\right\|_{H}, v_{1}^{\star}=\left\|\mathcal{A}^{1 / 2} v_{1}\right\|_{H}$ and $q^{\star}=\sup _{t \in[0, T]}\left\|\mathcal{A}^{1 / 2} q(t)\right\|_{H}$. Then, the solution map (1) for the IVP (21) has a unique fixed point in $B\left(v_{0}, R\right)$.

Proof. Let define the map

$$
\Phi: \mathcal{C} \rightarrow \mathcal{C} \text { with } \Phi(v(t))=\sum_{i=1}^{5} \Phi_{i}(v(t))
$$

where $v \in \mathcal{C}$ and $\Phi_{i}(v(t)), i=1,2, \ldots, 5$, are the five terms of (1). To show that $\Phi$ is well defined, we should prove first that $\Phi(v(t)), t \in[0, T]$, is a $D(\mathcal{A})$-valued function, which is continuous on 
$t \in[0, T]$ under the $\mathcal{C}$-norm, see $(25)$. As a second step, we have to prove that $\Phi$ satisfies the assertions of Theorem 2.2 in [22].

Using the results of Proposition 1 in [25] and Lemma 1 , since $\xi_{0} \in D(\mathcal{A})$ and $\xi_{1} \in D\left(\mathcal{A}^{1 / 2}\right)$, we obtain that

$$
\Phi_{1}(v(t))=(C(t)+S(t) \mathcal{B}) \xi_{0} \in D(\mathcal{A})
$$

and that

$$
\Phi_{2}(v(t))=S(t) \xi_{1} \in D(\mathcal{A}), t \in[0, T] .
$$

Relying next on the commutation of $\mathcal{A}^{1 / 2}\left(\right.$ on $\left.D\left(\mathcal{A}^{1 / 2}\right)\right)$ and $\mathcal{A}($ on $D(\mathcal{A}))$ for both $C(t)$ and $S(t), t \in$ $[0, T]$, on the strong continuity of $C(t)$ and $S(t) \mathcal{B}$ on $t \in[0, T]$, and on the fact that the function $\mathcal{A}^{1 / 2} S(t) \mathcal{A}^{1 / 2} \xi_{1}$ is continuous on $t \in[0, T]$ for $\xi_{1} \in D\left(\mathcal{A}^{1 / 2}\right)$ leads to

$$
\begin{gathered}
\left\|[\mathcal{A} C(t+h)-\mathcal{A} C(t)] \xi_{0}+[\mathcal{A} S(t+h) \mathcal{B}-\mathcal{A} S(t) \mathcal{B}] \xi_{0}\right\|_{H} \leq \\
\left\|[C(t+h)-C(t)] \mathcal{A} \xi_{0}\right\|_{H}+\left\|[S(t+h)-S(t)] \mathcal{B} \mathcal{A} \xi_{0}\right\|_{H} \rightarrow 0, \text { as }|h| \rightarrow 0
\end{gathered}
$$

and

$$
\left\|[\mathcal{A} S(t+h)-\mathcal{A} S(t)] \xi_{1}\right\|_{H}=\left\|\left[\mathcal{A}^{1 / 2} S(t+h)-\mathcal{A}^{1 / 2} S(t)\right] \mathcal{A}^{1 / 2} \xi_{1}\right\|_{H} \rightarrow 0, \quad \text { as } \quad|h| \rightarrow 0 .
$$

Next, we show that

$$
\Phi_{3}(v(t))=-\int_{0}^{t} C(t-s) \mathcal{B} v(s) d s \in D(\mathcal{A}), t \in[0, T]
$$

Since $v(t) \in D(\mathcal{A}), t \in[0, T]$, and $\mathcal{B}$ is a multiplicative operator (hence $\mathcal{B} v(t) \in D(\mathcal{A}), t \in[0, T]$ ), we obtain that $C(t-s) \mathcal{B} v(s) \in D(\mathcal{A})$ for $s, t \in[0, T]$. Further, since $\|C(t)\|_{\mathcal{L}(H)} \leq 1$ for $t \in \mathbb{R}$, we have that

$$
\begin{gathered}
\left\|\int_{0}^{t} \mathcal{A} C(t-s) \mathcal{B} v(s) d s\right\|_{H}=\left\|\int_{0}^{t} C(t-s) \mathcal{B} \mathcal{A} v(s) d s\right\|_{H} \leq \int_{0}^{t}\|C(t-s) \mathcal{B} \mathcal{A} v(s)\|_{H} d s \leq \\
\int_{0}^{t}\|C(t-s) \mathcal{B}\|_{\mathcal{L}(H)}\|\mathcal{A} v(s)\|_{H} d s \leq T \frac{c}{\rho A} \sup _{t \in[0, T]}\|v(t)\|_{H_{\mathcal{A}}}<\infty .
\end{gathered}
$$

Thus, $\Phi_{3}(v(t)) \in D(\mathcal{A})$ and $\mathcal{A}\left(\int_{0}^{t} C(t-s) \mathcal{B} v(s) d s\right)=\int_{0}^{t} \mathcal{A} C(t-s) \mathcal{B} v(s) d s$, by the closedness of $\mathcal{A}$. Moreover,

$$
\begin{gathered}
\left\|\int_{0}^{t+h} \mathcal{A} C(t+h-s) \mathcal{B} v(s) d s-\int_{0}^{t} \mathcal{A} C(t-s) \mathcal{B} v(s) d s\right\|_{H}= \\
\left\|\int_{0}^{t} \mathcal{A}[C(t+h-s)-C(t-s)] \mathcal{B} v(s) d s+\int_{t}^{t+h} \mathcal{A} C(t+h-s) \mathcal{B} v(s) d s\right\|_{H} \leq \\
\int_{0}^{t}\|[C(t+h-s)-C(t-s)] \mathcal{B} \mathcal{A} v(s)\|_{H} d s+\int_{t}^{t+h}\|C(t+h-s) \mathcal{B} \mathcal{A} v(s)\|_{H} d s \leq
\end{gathered}
$$




$$
\int_{0}^{t}\|[C(t+h-s)-C(t-s)] \mathcal{B} \mathcal{A} v(s)\|_{H} d s+h \frac{c}{\rho A} \sup _{t \in[0, T]}\|v(t)\|_{H_{\mathcal{A}}} \rightarrow 0, \text { as }|h| \rightarrow 0,
$$

by the Lebesgue dominated convergence theorem and the strong continuity of $C(t), t \in[0, T]$. Therefore, $\Phi_{3}(v(t)) t \in[0, T]$ is continuous under the $\mathcal{C}$-norm. For the nonlinear term

$$
\Phi_{4}(v(t))=-\frac{A}{2 L I} \int_{0}^{t} S(t-s)<\mathcal{A}^{1 / 2} v(s), v(s)>_{H} \mathcal{A}^{1 / 2} v(s) d s, t \in[0, T]
$$

as mentioned previously, if $v(t) \in D(\mathcal{A}), t \in[0, T]$, then

$$
S(t-s)<\mathcal{A}^{1 / 2} v(s), v(s)>_{H} \mathcal{A}^{1 / 2} v(s) \in D(\mathcal{A}) \text {, for } s, t \in[0, T] .
$$

By Lemmas 1 and 2, we obtain that

$$
\begin{gathered}
\left\|\int_{0}^{t} \mathcal{A} S(t-s)<\mathcal{A}^{1 / 2} v(s), v(s)>_{H} \mathcal{A}^{1 / 2} v(s) d s\right\|_{H} \\
=\left\|\int_{0}^{t} \mathcal{A}^{1 / 2} S(t-s)<\mathcal{A}^{1 / 2} v(s), v(s)>_{H} \mathcal{A} v(s) d s\right\|_{H} \\
\leq \int_{0}^{t}\left\|<\mathcal{A}^{1 / 2} v(s), v(s)>_{H} \mathcal{A} v(s)\right\|_{H} d s \\
\leq \int_{0}^{t}\left|<\mathcal{A}^{1 / 2} v(s), v(s)>_{H}\right|\|\mathcal{A} v(s)\|_{H} d s \\
\leq \int_{0}^{t}\left\|\mathcal{A}^{1 / 4} v(s)\right\|_{H}^{2}\|\mathcal{A} v(s)\|_{H} d s \\
\leq T M_{\mathcal{A}}^{2}\left(\sup _{t \in[0, T]}\|v(t)\|_{H_{\mathcal{A}}}\right)^{3}<\infty
\end{gathered}
$$

Hence, $\Phi_{4}(v(t)) \in D(\mathcal{A}), t \in[0, T]$. Further,

$$
\begin{gathered}
\| \int_{0}^{t+h} \mathcal{A} S(t+h-s)<\mathcal{A}^{1 / 2} v(s), v(s)>_{H} \mathcal{A}^{1 / 2} v(s) d s \\
-\int_{0}^{t} \mathcal{A} S(t-s)<\mathcal{A}^{1 / 2} v(s), v(s)>_{H} \mathcal{A}^{1 / 2} v(s) d s \|_{H}= \\
\| \int_{0}^{t}\left[\mathcal{A}^{1 / 2} S(t+h-s)-\mathcal{A}^{1 / 2} S(t-s)\right]<\mathcal{A}^{1 / 2} v(s), v(s)>_{H} \mathcal{A} v(s) d s+ \\
\int_{t}^{t+h} \mathcal{A}^{1 / 2} S(t+h-s)<\mathcal{A}^{1 / 2} v(s), v(s)>_{H} \mathcal{A} v(s) d s \|_{H} \leq \\
\int_{0}^{t}\left\|\left[\mathcal{A}^{1 / 2} S(t+h-s)-\mathcal{A}^{1 / 2} S(t-s)\right]<\mathcal{A}^{1 / 2} v(s), v(s)>_{H} \mathcal{A} v(s)\right\|_{H} d s \\
+h M_{\mathcal{A}}^{2}\left(\sup _{t \in[0, T]}\|v(t)\|_{H_{\mathcal{A}}}\right)^{3} \rightarrow 0, \text { as }|h| \rightarrow 0 .
\end{gathered}
$$


Considering the Lebesgue dominated convergence theorem, the function $\mathcal{A}^{1 / 2} S(t) y$ for $y \in H$ is continuous on $t \in[0, T]$. Therefore, $\Phi_{4}(v(t)), t \in[0, T]$, is continuous under the $\mathcal{C}$-norm. For

$$
\Phi_{5}(v(t))=\frac{1}{\rho A} \int_{0}^{t} S(t-s) q(s) d s
$$

it is observed that since $q(t) \in D\left(\mathcal{A}^{1 / 2}\right), t \in[0, T]$, then $S(t-s) q(s) \in D(\mathcal{A})$, for $s, t \in[0, T]$. Furthermore, by Lemma 1 and assumptions, (26) and (27), we obtain that

$$
\left\|\int_{0}^{t} \mathcal{A} S(t-s) q(s) d s\right\|_{H} \leq \int_{0}^{t}\left\|\mathcal{A}^{1 / 2} S(t-s) \mathcal{A}^{1 / 2} q(s)\right\|_{H} d s \leq \int_{0}^{T}\|q(t)\|_{H_{\mathcal{A}^{1 / 2}}} d t<\infty .
$$

Hence, $\Phi_{5}(v(t)) \in D(\mathcal{A}), t \in[0, T]$, and

$$
\begin{gathered}
\left\|\int_{0}^{t+h} \mathcal{A}^{1 / 2} S(t+h-s) \mathcal{A}^{1 / 2} q(s) d s-\int_{0}^{t} \mathcal{A}^{1 / 2} S(t-s) \mathcal{A}^{1 / 2} q(s) d s\right\|_{H}= \\
\left\|\int_{0}^{t}\left[\mathcal{A}^{1 / 2} S(t+h-s)-\mathcal{A}^{1 / 2} S(t-s)\right] \mathcal{A}^{1 / 2} q(s) d s+\int_{t}^{t+h} \mathcal{A}^{1 / 2} S(t+h-s) \mathcal{A}^{1 / 2} q(s) d s\right\|_{H} \leq \\
\int_{0}^{t}\left\|\left[\mathcal{A}^{1 / 2} S(t+h-s)-\mathcal{A}^{1 / 2} S(t-s)\right] \mathcal{A}^{1 / 2} q(s)\right\|_{H} d s+h \int_{0}^{T}\left\|\mathcal{A}^{1 / 2} q(t)\right\|_{H} d t \rightarrow 0, \text { as }|h| \rightarrow 0,
\end{gathered}
$$

by the Lebesgue dominated convergence theorem, since $\mathcal{A}^{1 / 2} S(t) \mathcal{A}^{1 / 2} y$, for $y \in \mathcal{A}^{1 / 2}$, is continuous on $t \in[0, T]$. Therefore, $\Phi_{5}(v(t)), t \in[0, T]$, is continuous under the $\mathcal{C}$-norm. In what follows, and to complete the proof, the necessary conditions are determined for $\Phi: B\left(v_{0}, R\right) \rightarrow \mathcal{C}$ to be a contraction. Let $u_{1}, u_{2} \in B\left(v_{0}, R\right)$, then we have that

$$
\left\|\Phi\left(u_{1}\right)-\Phi\left(u_{2}\right)\right\|_{\mathcal{C}} \leq\left\|\Phi_{3}\left(u_{1}\right)-\Phi_{3}\left(u_{2}\right)\right\|_{\mathcal{C}}+\left\|\Phi_{4}\left(u_{1}\right)-\Phi_{4}\left(u_{2}\right)\right\|_{\mathcal{C}}
$$

For the term $\left\|\Phi_{3}\left(u_{1}\right)-\Phi_{3}\left(u_{2}\right)\right\|_{\mathcal{C}}$ in (30), it is observed that

$$
\begin{gathered}
\left\|\int_{0}^{t} \mathcal{A} C(t-s) \mathcal{B}\left[u_{1}(s)-u_{2}(s)\right] d s\right\|_{H} \leq \int_{0}^{t}\left\|C(t-s) \mathcal{B}\left[\mathcal{A} u_{1}(s)-\mathcal{A} u_{2}(s)\right]\right\|_{H} d s \\
\leq \int_{0}^{t}\|C(t-s) \mathcal{B}\|_{\mathcal{L}(H)}\left\|\left[\mathcal{A} u_{1}(s)-\mathcal{A} u_{2}(s)\right]\right\|_{H} d s \\
\leq T \frac{c}{\rho A} \sup _{t \in[0, T]}\left\|\mathcal{A} u_{1}(t)-\mathcal{A} u_{2}(t)\right\|_{H} .
\end{gathered}
$$

Thus, the following expression is derived, i.e.,

$$
\left\|\Phi_{3}\left(u_{1}\right)-\Phi_{3}\left(u_{2}\right)\right\|_{\mathcal{C}} \leq T \sqrt{1+M_{\mathcal{A}}^{2}} \frac{c}{\rho A}\left\|u_{1}-u_{2}\right\|_{\mathcal{C}} .
$$

Regarding the term $\left\|\Phi_{4}\left(u_{1}\right)-\Phi_{4}\left(u_{2}\right)\right\|_{\mathcal{C}}$ in $(30)$, we find that:

$$
\left\|\int_{0}^{t} \mathcal{A} S(t-s)\left[<\mathcal{A}^{1 / 2} u_{1}(s), u_{1}(s)>_{H} \mathcal{A}^{1 / 2} u_{1}(s)-<\mathcal{A}^{1 / 2} u_{2}(s), u_{2}(s)>_{H} \mathcal{A}^{1 / 2} u_{2}(s)\right] d s\right\|_{H}
$$




$$
\begin{aligned}
& \leq \int_{0}^{t} \| \mathcal{A}^{1 / 2} S(t-s)\left[<\mathcal{A}^{1 / 2} u_{1}(s), u_{1}(s)>_{H} \mathcal{A} u_{1}(s)-<\mathcal{A}^{1 / 2} u_{2}(s), u_{2}(s)>_{H} \mathcal{A} u_{2}(s) \|_{H}\right] d s \leq \\
& \int_{0}^{t}\left\|<\mathcal{A}^{1 / 2} u_{1}(s), u_{1}(s)>_{H}\left(\mathcal{A} u_{1}(s)-\mathcal{A} u_{2}(s)\right)+\left(<\mathcal{A}^{1 / 2} u_{1}(s), u_{1}(s)>_{H}-<\mathcal{A}^{1 / 2} u_{2}(s), u_{2}(s)>_{H}\right) \mathcal{A} u_{2}(s)\right\|_{H} d s \\
& \leq \int_{0}^{t}\left|<\mathcal{A}^{1 / 2} u_{1}(s), u_{1}(s)>_{H}\right|\left\|\mathcal{A} u_{1}(s)-\mathcal{A} u_{2}(s)\right\|_{H} d s \\
& +\int_{0}^{t}\left|<\mathcal{A}^{1 / 2} u_{1}(s), u_{1}(s)>_{H}-<\mathcal{A}^{1 / 2} u_{2}(s), u_{2}(s)>_{H}\right|\left\|\mathcal{A} u_{2}(s)\right\|_{H} d s \\
& =\int_{0}^{t}\left\|\mathcal{A}^{1 / 4} u_{1}(s)\right\|_{H}^{2}\left\|\mathcal{A} u_{1}(s)-\mathcal{A} u_{2}(s)\right\|_{H} d s \\
& +\int_{0}^{t}\left|\left\|\mathcal{A}^{1 / 4} u_{1}(s)\right\|_{H}^{2}-\left\|\mathcal{A}^{1 / 4} u_{2}(s)\right\|_{H}^{2}\right|\left\|\mathcal{A} u_{2}(s)\right\|_{H} d s \\
& \leq \int_{0}^{t}\left\|\mathcal{A}^{1 / 4} u_{1}(s)\right\|_{H}^{2} d s \sup _{t \in[0, T]}\left\|\mathcal{A} u_{1}(t)-\mathcal{A} u_{2}(t)\right\|_{H}+ \\
& \int_{0}^{t}\left|\left\|\mathcal{A}^{1 / 4} u_{1}(s)\right\|_{H}-\left\|\mathcal{A}^{1 / 4} u_{2}(s)\right\|_{H}\right|\left(\left\|\mathcal{A}^{1 / 4} u_{1}(s)\right\|_{H}+\left\|\mathcal{A}^{1 / 4} u_{2}(s)\right\|_{H}\right)\left\|\mathcal{A} u_{2}(s)\right\|_{H} d s \\
& \leq \int_{0}^{t} d s\left(\sup _{t \in[0, T]}\left\|\mathcal{A}^{1 / 4} u_{1}(t)\right\|_{H}\right)^{2} \sup _{t \in[0, T]}\left\|\mathcal{A} u_{1}(t)-\mathcal{A} u_{2}(t)\right\|_{H} \\
& +\int_{0}^{t} d s\left[\sup _{t \in[0, T]}\left(\left\|\mathcal{A}^{1 / 4} u_{1}(t)\right\|_{H}+\left\|\mathcal{A}^{1 / 4} u_{2}(t)\right\|_{H}\right)\right] \sup _{t \in[0, T]}\left\|\mathcal{A} u_{2}(t)\right\|_{H} \\
& \times \sup _{t \in[0, T]}\left\|\mathcal{A}^{1 / 4} u_{1}(t)-\mathcal{A}^{1 / 4} u_{2}(t)\right\|_{H} .
\end{aligned}
$$

Next, applying Lemma 2 yields

$$
\begin{gathered}
\left\|\int_{0}^{t} \mathcal{A} S(t-s)\left[<\mathcal{A}^{1 / 2} u_{1}(s), u_{1}(s)>_{H} \mathcal{A}^{1 / 2} u_{1}(s)-<\mathcal{A}^{1 / 2} u_{2}(s), u_{2}(s)>_{H} \mathcal{A}^{1 / 2} u_{2}(s)\right] d s\right\|_{H} \\
\leq T M_{\mathcal{A}}^{2}\left[\left(\sup _{t \in[0, T]}\left\|\mathcal{A} u_{1}(t)\right\|_{H}\right)^{2}+\sup _{t \in[0, T]}\left(\left\|\mathcal{A} u_{1}(t)\right\|_{H}+\left\|\mathcal{A} u_{2}(t)\right\|_{H}\right) \sup _{t \in[0, T]}\left\|\mathcal{A} u_{2}(t)\right\|_{H}\right] \\
\times \sup _{t \in[0, T]}\left\|\mathcal{A} u_{1}(t)-\mathcal{A} u_{2}(t)\right\|_{H} \leq \\
T M_{\mathcal{A}}^{2}\left[\left(\left\|u_{1}-v_{0}\right\|_{\mathcal{C}}+\left\|v_{0}\right\|_{\mathcal{C}}\right)^{2}+\left(\left\|u_{1}-v_{0}\right\|_{\mathcal{C}}+\left\|u_{2}-v_{0}\right\|_{\mathcal{C}}+2\left\|v_{0}\right\|_{\mathcal{C}}\right)\left(\left\|u_{2}-v_{0}\right\|_{\mathcal{C}}+\left\|v_{0}\right\|_{\mathcal{C}}\right)\right] \\
\times\left\|u_{1}-u_{2}\right\|_{\mathcal{C}}= \\
T M_{\mathcal{A}}^{2}\left[\left(R+v_{0}^{\star}\right)^{2}+\left(2 R+2 v_{0}^{\star}\right)\left(R+v_{0}^{\star}\right)\right] \times\left\|u_{1}-u_{2}\right\|_{\mathcal{C}}= \\
3 T M_{\mathcal{A}}^{2}\left(R+v_{0}^{\star}\right)^{2} \times\left\|u_{1}-u_{2}\right\|_{\mathcal{C} .}
\end{gathered}
$$


Thus, $\Phi_{4}: B\left(v_{0}, R\right) \rightarrow \mathcal{C}$ is a contraction under the condition

$$
T \sqrt{1+M_{\mathcal{A}}^{2}} \frac{3 A}{2 L I} M_{\mathcal{A}}^{2}\left(R+v_{0}^{\star}\right)^{2}<1 .
$$

Combining (31) and (32), it is proved that $\Phi: B\left(v_{0}, R\right) \rightarrow \mathcal{C}$ is a contraction under the condition

$$
h=T \sqrt{1+M_{\mathcal{A}}^{2}}\left[\frac{c}{\rho A}+\frac{3 A}{2 L I} M_{\mathcal{A}}^{2}\left(R+v_{0}^{\star}\right)^{2}\right]<1 .
$$

Therefore, the relation (28) in Theorem 1 is derived. Next, the conditions such that

$$
\left\|\Phi\left(v_{0}\right)-v_{0}\right\|_{\mathcal{C}}<R(1-h)
$$

are sought for. In this regard,

$$
\begin{gathered}
\left\|\mathcal{A} \Phi\left(v_{0}\right)-\mathcal{A} v_{0}\right\|_{H} \leq \\
\left\|(C(t)+S(t) \mathcal{B}-I) \mathcal{A} v_{0}\right\|_{H}+\left\|\mathcal{A} S(t) v_{1}\right\|_{H}+\left\|\int_{0}^{t} C(t-s) \mathcal{B} \mathcal{A} v_{0} d s\right\|_{H} \\
+\frac{A}{2 L I}\left\|\int_{0}^{t} \mathcal{A} S(t-s)<\mathcal{A}^{1 / 2} v_{0}, v_{0}>_{H} \mathcal{A}^{1 / 2} v_{0} d s\right\|_{H}+\frac{1}{\rho A}\left\|\int_{0}^{t} \mathcal{A} S(t-s) q(s) d s\right\|_{H} \leq \\
\left(2+\frac{c M_{S}}{\rho A}\right)\left\|\mathcal{A} v_{0}\right\|_{H}+\left\|\mathcal{A}^{1 / 2} v_{1}\right\|_{H}+\frac{c T}{\rho A}\left\|\mathcal{A} v_{0}\right\|_{H}+\frac{T A}{2 L I}\left|<\mathcal{A}^{1 / 2} v_{0}, v_{0}>_{H}\right|\left\|\mathcal{A} v_{0}\right\|+\frac{T}{\rho A}\left\|\mathcal{A}^{1 / 2} q(s)\right\|_{H} \\
\leq\left(2+\frac{c M_{S}}{\rho A}\right) v_{0}^{\star}+v_{1}^{\star}+\frac{c T}{\rho A} v_{0}^{\star}+\frac{T A}{2 L I} M_{\mathcal{A}}^{2}\left(v_{0}^{\star}\right)^{3}+\frac{T}{\rho A} q^{\star} .
\end{gathered}
$$

Therefore, we obtain that

$$
\left\|\Phi\left(v_{0}\right)-v_{0}\right\|_{\mathcal{C}}<\sqrt{1+M_{\mathcal{A}}^{2}}\left[\left(2+\frac{c\left(M_{S}+T\right)}{\rho A}\right) v_{0}^{\star}+v_{1}^{\star}+\frac{T A}{2 L I} M_{\mathcal{A}}^{2}\left(v_{0}^{\star}\right)^{3}+\frac{T}{\rho A} q^{\star}\right]
$$

Combining now expressions (33) and (34), the relation (29) in Theorem 1 is derived.

In order to express the solutions maps as functions from $[0, T] \times[0, L]$ to $\mathbb{R}$, without involving any spatial derivatives, the following result with respect to the nonlinearity term invoked.

Lemma 3. Let $v(s) \in D\left(\mathcal{A}^{1 / 2}\right), s \in[0, T]$. Then, the term of the solution map which contains the nonlinear term, considered as a function from $[0, T] \times[0, L]$ to $\mathbb{R}$, is expressed as

$$
\begin{gathered}
\frac{A}{2 L I} \int_{0}^{t} S(t-s)<\mathcal{A}^{1 / 2} v(s), v(s)>_{H} \mathcal{A}^{1 / 2} v(s) d s= \\
\frac{2 \pi^{2}}{L^{5}} \sqrt{\frac{E A}{\rho I}} \int_{0}^{t}\left\{\sum_{n=1}^{\infty}\left[n^{2}\left(\int_{0}^{L} v(s, z) \sin \left(\frac{n \pi}{L} z\right) d z\right)^{2}\right] \times\right. \\
\left.\sum_{n=1}^{\infty}\left[\sin \left[\sqrt{\frac{E I}{\rho A}} \frac{n^{2} \pi^{2}}{L^{2}}(t-s)\right]\left(\int_{0}^{L} v(s, z) \sin \left(\frac{n \pi}{L} z\right) d z\right) \sin \left(\frac{n \pi}{L} x\right)\right]\right\} d s .
\end{gathered}
$$


Proof. Similar computations as in the proof of Lemma 1 in [25] are provided with respect to the family $S(t) \mathcal{A}^{1 / 2}, t \in \mathbb{R}$. Thus, we obtain that

$$
\begin{gathered}
S(t-s) \mathcal{A}^{1 / 2} v(s)=\sum_{n=1}^{\infty} \sin \left[\sqrt{\frac{E I}{\rho A}} \frac{n^{2} \pi^{2}}{L^{2}}(t-s)\right]<v(s), e_{n}>_{H} e_{n}= \\
\frac{2}{L} \sum_{n=1}^{\infty} \sin \left[\sqrt{\frac{E I}{\rho A}} \frac{n^{2} \pi^{2}}{L^{2}}(t-s)\right]\left(\int_{0}^{L} v(s, z) \sin \left(\frac{n \pi}{L} z\right) d z\right) \sin \left(\frac{n \pi}{L} x\right), \quad s, t \in[0, T], x \in[0, L] .
\end{gathered}
$$

Furthermore, by the spectral representation of $\mathcal{A}^{1 / 2}$, we have that

$$
\begin{gathered}
\left.<\mathcal{A}^{1 / 2} v(s), v(s)\right\rangle_{H}=\left\langle\sum_{n=1}^{\infty} \sqrt{\frac{E I}{\rho A}} \frac{n^{2} \pi^{2}}{L^{2}}<v(s), e_{n}>_{H} e_{n}, \sum_{m=1}^{\infty}\left\langle v(s), e_{m}>_{H} e_{m}\right\rangle_{H}=\right. \\
\sum_{n=1}^{\infty} \sqrt{\frac{E I}{\rho A}} \frac{n^{2} \pi^{2}}{L^{2}}\left|<v(s), e_{n}>_{H}\right|^{2}=\sum_{n=1}^{\infty}\left[\sqrt{\frac{E I}{\rho A}} \frac{n^{2} \pi^{2}}{L^{2}} \frac{2}{L}\left(\int_{0}^{L} v(s, z) \sin \left(\frac{n \pi}{L} z\right) d z\right)^{2}\right]= \\
\frac{2 \pi^{2}}{L^{3}} \sqrt{\frac{E I}{\rho A}} \sum_{n=1}^{\infty}\left[n^{2}\left(\int_{0}^{L} v(s, z) \sin \left(\frac{n \pi}{L} z\right) d z\right)^{2}\right] .
\end{gathered}
$$

Finally, returning to the initial problem (2), we obtain an implicit analytic expression for the solution $v(t, x)$, Eq. (3). However, prior to presenting it, the following expressions are defined:

$$
\begin{gathered}
v_{1}(t, x):=\frac{2}{L} \sum_{n=1}^{\infty}\left[\cos \left(\sqrt{\frac{E I}{\rho A}} \frac{n^{2} \pi^{2}}{L^{2}} t\right)\left(\int_{0}^{L} v_{0}(z) \sin \left(\frac{n \pi}{L} z\right) d z\right) \sin \left(\frac{n \pi}{L} x\right)\right] \\
v_{2}(t, x):=\frac{2 c L}{\pi^{2} \sqrt{E I \rho A}} \sum_{n=1}^{\infty}\left[\frac{1}{n^{2}} \sin \left(\sqrt{\frac{E I}{\rho A}} \frac{n^{2} \pi^{2}}{L^{2}} t\right)\left(\int_{0}^{L} v_{0}(z) \sin \left(\frac{n \pi}{L} z\right) d z\right) \sin \left(\frac{n \pi}{L} x\right)\right] \\
v_{3}(t, x):=\frac{2 L}{\pi^{2}} \sqrt{\frac{\rho A}{E I}} \sum_{n=1}^{\infty}\left[\frac{1}{n^{2}} \sin \left(\sqrt{\frac{E I}{\rho A}} \frac{n^{2} \pi^{2}}{L^{2}} t\right)\left(\int_{0}^{L} v_{1}(z) \sin \left(\frac{n \pi}{L} z\right) d z\right) \sin \left(\frac{n \pi}{L} x\right)\right] \\
v_{4}(t, x):=\frac{2 c}{L \rho A} \int_{0}^{t}\left\{\sum_{n=1}^{\infty}\left[\cos \left(\sqrt{\frac{E I}{\rho A}} \frac{n^{2} \pi^{2}}{L^{2}}(t-s)\right)\left(\int_{0}^{L} v(s, z) \sin \left(\frac{n \pi}{L} z\right) d z\right) \sin \left(\frac{n \pi}{L} x\right)\right]\right\} d s, \\
v_{5}(t, x):=\frac{2 \pi^{2}}{L^{5}} \sqrt{\frac{E A}{\rho I}} \int_{0}^{t}\left\{\sum_{n=1}^{\infty}\left[n^{2}\left(\int_{0}^{L} v(s, z) \sin \left(\frac{n \pi}{L} z\right) d z\right)^{2}\right],\right. \\
v_{7}(t, x):=\frac{2 L}{\pi^{2} \sqrt{E I \rho A}} \int_{0}^{\infty}\left\{\sum_{n=1}^{\infty}\left[\frac{1}{n^{2}} \sin \left[\sqrt{\frac{E I}{\rho A}} \frac{n^{2} \pi^{2}}{L^{2}}(t-s)\right]\left(\int_{0}^{L} v(s, z) \sin \left(\frac{n \pi}{L} z\right) d z\right) \sin \left(\frac{n \pi}{L} x\right)\right]\right\} d s \\
\left.\left.\left.\frac{E I}{\rho A} \frac{n^{2} \pi^{2}}{L^{2}}(t-s)\right)\left(\int_{0}^{L} q(s, z) \sin \left(\frac{n \pi}{L} z\right) d z\right) \sin \left(\frac{n \pi}{L} x\right)\right]\right\} d s .
\end{gathered}
$$


Thus, by employing Eqs. (35) - (41), the implicit analytic solution $v(t, x)$ is provided by

$$
v(t, x)=\sum_{k=1}^{3} v_{k}(t, x)-v_{4}(t, x)-v_{5}(t, x) \times v_{6}(t, x)+v_{7}(t, x) .
$$

\section{$2.2 \quad$ Viscoelastic case}

Adopting the analysis of Section 2.1, the IBVP (1) obtains the form of a second order nonlinear ODE in the Hilbert space $H$, which now involves a fractional derivative term as well:

$$
\begin{gathered}
v^{\prime \prime}(t)+\frac{c}{\rho A}{ }_{0} D_{t}^{\alpha} v(t)+\mathcal{A} v(t)+\frac{A}{2 L I}<\mathcal{A}^{1 / 2} v(t), v(t)>_{H} \mathcal{A}^{1 / 2} v(t)=\frac{1}{\rho A} q(t), \text { a.e. on }(0, T), \\
v(0)=v_{0}, v^{\prime}(0)=v_{1} .
\end{gathered}
$$

The IVP (43) admits a unique solution, i.e.,

$$
\begin{aligned}
v(t)=C(t) v_{0}+S(t) v_{1}-\frac{c}{\rho A \Gamma(1-\alpha)} \int_{0}^{t} S(t-s) \int_{0}^{s} \frac{v^{\prime}(r)}{(s-r)^{\alpha}} d r d s \\
\quad-\frac{A}{2 L I} \int_{0}^{t} S(t-s)<\mathcal{A}^{1 / 2} v(s), v(s)>_{H} \mathcal{A}^{1 / 2} v(s) d s+\frac{1}{\rho A} \int_{0}^{t} S(t-s) q(s) d s, \quad t \in[0, T] .
\end{aligned}
$$

Furthermore, to treat the fractional derivative term, and obtain a derivative-free expression, the following important result is proved:

Lemma 4. Assume that $v \in \mathcal{C}$ and that $v^{\prime}(t) \frac{1}{t^{\alpha}} \in L^{1}(0, T ; H)$. Then, the following expressions hold; that is,

1.

$$
\int_{0}^{t} S(t-s) \int_{0}^{s} \frac{v^{\prime}(r)}{(s-r)^{\alpha}} d r d s=\int_{0}^{t}\left(\int_{0}^{s} C(s-r) v(r) d r-S(s) v(0)\right) \frac{1}{(t-s)^{\alpha}} d s
$$

2.

$$
\frac{d}{d t}\left(\int_{0}^{t} S(t-s) \int_{0}^{s} \frac{v^{\prime}(r)}{(s-r)^{\alpha}} d r d s\right)=\int_{0}^{t}\left(-\int_{0}^{s} \mathcal{A} S(s-r) v(r) d r+v(s)-C(s) v(0)\right) \frac{1}{(t-s)^{\alpha}} d s .
$$

3. If also $v^{\prime} \in L^{1}\left(0, T ; H_{\mathcal{A}^{1 / 2}}\right)$, then

$$
\frac{d^{2}}{d t^{2}}\left(\int_{0}^{t} S(t-s) \int_{0}^{s} \frac{v^{\prime}(r)}{(s-r)^{\alpha}} d r d s\right)=\int_{0}^{t}\left(v^{\prime}(s)-\int_{0}^{s} \mathcal{A} S(s-r) v^{\prime}(r) d r\right) \frac{1}{(t-s)^{\alpha}} d s .
$$


Proof. Note that

$$
\int_{0}^{t} S(t-s) \int_{0}^{s} \frac{v^{\prime}(r)}{(s-r)^{\alpha}} d r d s=S(t) *\left(\frac{1}{t^{\alpha}} * v^{\prime}(t)\right)
$$

where $*$ denotes the convolution $f(t) * g(t)=\int_{0}^{t} f(t-s) g(s) d s$. Results 1 and 2 have been proved in [25]. For the Result 3, the differentiation and properties of the convolution are used. Thus, we observe that

$$
\begin{gathered}
\frac{d^{2}}{d t^{2}}\left(\int_{0}^{t} S(t-s) \int_{0}^{s} \frac{v^{\prime}(r)}{(s-r)^{\alpha}} d r d s\right)=\frac{d}{d t}\left(\int_{0}^{t} C(t-s) \int_{0}^{s} \frac{v^{\prime}(r)}{(s-r)^{\alpha}} d r d s\right) \\
=-\int_{0}^{t} \mathcal{A} S(t-s) \int_{0}^{s} \frac{v^{\prime}(r)}{(s-r)^{\alpha}} d r d s+\int_{0}^{t} \frac{v^{\prime}(s)}{(t-s)^{\alpha}} d s= \\
-\mathcal{A} S(t) *\left(\frac{1}{t^{\alpha}} * v^{\prime}(t)\right)+\int_{0}^{t} \frac{v^{\prime}(s)}{(t-s)^{\alpha}} d s=-\frac{1}{t^{\alpha}} *\left(\mathcal{A} S(t) * v^{\prime}(t)\right)+\int_{0}^{t} \frac{v^{\prime}(s)}{(t-s)^{\alpha}} d s \\
=\int_{0}^{t}\left(v^{\prime}(s)-\int_{0}^{s} \mathcal{A} S(s-r) v^{\prime}(r) d r\right) \frac{1}{(t-s)^{\alpha}} d s,
\end{gathered}
$$

which is well defined under the condition $v^{\prime} \in L^{1}\left(0, T ; H_{\mathcal{A}^{1 / 2}}\right)$.

Definition 2. A twice differentiable function $v:[0, T] \rightarrow H$, is called a solution of the IVP (21) if (i) $v(t) \in D(\mathcal{A}), \quad t \in[0, T]$,

(ii) $v(t)=C(t) v_{0}+S(t) v_{1}-\frac{c}{\rho A \Gamma(1-\alpha)} \int_{0}^{t}\left(\int_{0}^{s} C(s-r) v(r) d r-S(s) v_{0}\right) \frac{1}{(t-s)^{\alpha}} d s$

$$
-\frac{A}{2 L I} \int_{0}^{t} S(t-s)<\mathcal{A}^{1 / 2} v(s), v(s)>_{H} \mathcal{A}^{1 / 2} v(s) d s+\frac{1}{\rho A} \int_{0}^{t} S(t-s) q(s) d s, \quad t \in[0, T] .
$$

Applying Lemma 4 (in particular, result 1) and similar techniques as in the proof of Theorem 1 of section 2.1, we prove the following theorem is proved:

Theorem 2. Suppose that assumptions (26) and (27) hold, and the coefficients $v_{0}, v_{1}, q$, satisfy the following relations

$$
\begin{gathered}
h=T \sqrt{1+M_{\mathcal{A}}^{2}}\left[\frac{c T^{1-\alpha}}{(1-\alpha) \rho A \Gamma(1-\alpha)}+\frac{3 A}{2 L I} M_{\mathcal{A}}^{2}\left(R+v_{0}^{\star}\right)^{2}\right]<1, \\
\left.\sqrt{1+M_{\mathcal{A}}^{2}}\left[\left(2+\frac{c T^{1-\alpha}\left(T+M_{S}\right)}{(1-\alpha) \rho A \Gamma(1-\alpha)}\right)\right) v_{0}^{\star}+v_{1}^{\star}+\frac{T A}{2 L I} M_{\mathcal{A}}^{2}\left(v_{0}^{\star}\right)^{3}+\frac{T}{\rho A} q^{\star}\right]+R h<R,
\end{gathered}
$$

for some $R>0$ and $T>0$, with $v_{0}^{\star}=\left\|\mathcal{A} v_{0}\right\|_{H}, v_{1}^{\star}=\left\|\mathcal{A}^{1 / 2} v_{1}\right\|_{H}$ and $q^{\star}=\sup _{t \in[0, T]}\left\|\mathcal{A}^{1 / 2} q(t)\right\|_{H}$. Then the solution map of Definition 2 for the IVP (43) has a unique fixed point in $B\left(v_{0}, R\right)$. 
Proof. For the solution map of Definition 2, attention is directed to the term which corresponds to the fractional derivative only, since all the other terms have been addressed in Theorem 1. It is first shown that

$$
\Phi_{3}(v(t))=\int_{0}^{t}\left(\int_{0}^{s} C(s-r) v(r) d r-S(s) v_{0}\right) \frac{1}{(t-s)^{\alpha}} d s \in D(\mathcal{A}),
$$

for $t \in[0, T]$. Since $v(t) \in D(\mathcal{A}), t \in[0, T]$, and $v_{0} \in D(\mathcal{A})$, we have that $C(s-r) v(r) \in D(\mathcal{A})$, for $r, s \in[0, T]$, and that $S(s) v_{0} \in D(\mathcal{A})$, for $s \in[0, T]$. Furthermore, considering $\|C(t)\|_{\mathcal{L}_{H}} \leq 1$, for $t \in \mathbb{R}$ yields

$$
\begin{gathered}
\left\|\int_{0}^{s} \mathcal{A} C(s-r) v(r) d r\right\|_{H}=\left\|\int_{0}^{s} C(s-r) \mathcal{A} v(r) d r\right\|_{H} \leq \int_{0}^{s}\|C(s-r) \mathcal{A} v(r)\|_{H} d r \\
\leq \int_{0}^{s}\|\mathcal{A} v(r)\|_{H} d r \leq T \sup _{t \in[0, T]}\|v(t)\|_{H_{\mathcal{A}}}<\infty .
\end{gathered}
$$

Thus, by the closedness of $\mathcal{A}$, we obtain that

$$
\mathcal{A}\left(\int_{0}^{s} C(s-r) v(r) d r\right)=\int_{0}^{s} \mathcal{A} C(s-r) v(r) d r, s \in[0, T]
$$

and that

$$
\mathcal{A}\left(\int_{0}^{s} C(s-r) v(r) d r-S(s) v_{0}\right)=\int_{0}^{s} C(s-r) \mathcal{A} v(r) d r-S(s) \mathcal{A} v_{0} s \in[0, T] .
$$

Moreover, applying standard arguments and properties of the convolution, we obtain in a straightforward manner that

$$
\begin{gathered}
\left\|\int_{0}^{t} \mathcal{A}\left(\int_{0}^{s} C(s-r) v(r) d r-S(s) v_{0}\right) \frac{1}{(t-s)^{\alpha}} d s\right\|_{H} \\
=\left\|\int_{0}^{t} \int_{0}^{s} C(s-r) \mathcal{A} v(r) d r \frac{1}{(t-s)^{\alpha}} d s-\int_{0}^{t} S(s) \mathcal{A} v_{0} \frac{1}{(t-s)^{\alpha}} d s\right\|_{H} \\
\leq\left\|\left[C(t) * \frac{1}{t^{\alpha}}\right] * \mathcal{A} v(t)\right\|_{H}+\int_{0}^{t}\left\|S(s) \mathcal{A} v_{0} \frac{1}{(t-s)^{\alpha}}\right\|_{H} d s \\
\leq \int_{0}^{t}\left\|\int_{0}^{s} C(s-r) \frac{1}{r^{\alpha}} d r \mathcal{A} v(t-s)\right\|_{H} d s+M_{S}\left\|\mathcal{A} v_{0}\right\|_{H} \int_{0}^{t} \frac{1}{s^{\alpha}} d s \\
\leq \int_{0}^{t}\left\|\int_{0}^{s} C(s-r) \frac{1}{r^{\alpha}} d r\right\|_{\mathcal{L}(H)}\|\mathcal{A} v(t-s)\|_{H} d s+M_{S}\left\|\mathcal{A} v_{0}\right\|_{H} \int_{0}^{t} \frac{1}{s^{\alpha}} d s \\
\leq \int_{0}^{t} \int_{0}^{s} \frac{1}{r^{\alpha}} d r\|\mathcal{A} v(t-s)\|_{H} d s+\left.M_{S}\left\|\mathcal{A} v_{0}\right\|_{H} \frac{s^{1-\alpha}}{1-\alpha}\right|_{0} ^{t} \\
\leq \frac{T^{2-\alpha}}{1-\alpha} \sup _{t \in[0, T]}\|\mathcal{A} v(t)\|_{H}+M_{S} \frac{T^{1-\alpha}}{1-\alpha}\left\|\mathcal{A} v_{0}\right\|_{H}<\infty .
\end{gathered}
$$


Hence, $\Phi_{3}(t) \in D(\mathcal{A})$, for $t \in[0, T]$. We will show now that $\Phi_{3}$ is continuous on $[0, T]$ under the $\mathcal{C}$-norm. By employing the properties of convolution and some direct calculations, we obtain that

$$
\begin{aligned}
& \left\|\mathcal{A} \Phi_{3}(t+h)-\mathcal{A} \Phi_{3}(t)\right\|_{H}= \\
& \| \int_{0}^{t+h}\left(\int_{0}^{s} C(s-r) \mathcal{A} v(r) d r-S(s) \mathcal{A} v_{0}\right) \frac{1}{(t+h-s)^{\alpha}} d s \\
& -\int_{0}^{t}\left(\int_{0}^{s} C(s-r) \mathcal{A} v(r) d r-S(s) \mathcal{A} v_{0}\right) \frac{1}{(t-s)^{\alpha}} d s \|_{H} \\
& \leq\left\|\int_{0}^{t}\left(\int_{0}^{s} C(s-r) \mathcal{A} v(r) d r-S(s) \mathcal{A} v_{0}\right)\left(\frac{1}{(t+h-s)^{\alpha}}-\frac{1}{(t-s)^{\alpha}}\right) d s\right\|_{H} \\
& +\left\|\int_{t}^{t+h}\left(\int_{0}^{s} C(s-r) \mathcal{A} v(r) d r-S(s) \mathcal{A} v_{0}\right) \frac{1}{(t+h-s)^{\alpha}} d s\right\|_{H}= \\
& \left\|\int_{0}^{t} \int_{0}^{s} C(s-r)\left(\frac{1}{(r+h)^{\alpha}}-\frac{1}{r^{\alpha}}\right) d r \mathcal{A} v(t-s) d s-\int_{0}^{t} S(s) \mathcal{A} v_{0}\left(\frac{1}{(t+h-s)^{\alpha}}-\frac{1}{(t-s)^{\alpha}}\right) d s\right\|_{H} \\
& +\left\|\int_{t}^{t+h} \int_{0}^{s} C(s-r) \frac{1}{(r+h)^{\alpha}} d r \mathcal{A} v(t-s) d s-\int_{t}^{t+h} S(s) \mathcal{A} v_{0} \frac{1}{(t+h-s)^{\alpha}} d s\right\|_{H} \\
& \leq\left\|\int_{0}^{t} \int_{0}^{s} C(s-r)\left(\frac{1}{(r+h)^{\alpha}}-\frac{1}{r^{\alpha}}\right) d r \mathcal{A} v(t-s) d s\right\|_{H} \\
& +\left\|\int_{0}^{t} S(s) \mathcal{A} v_{0}\left(\frac{1}{(t+h-s)^{\alpha}}-\frac{1}{(t-s)^{\alpha}}\right) d s\right\|_{H} \\
& +\left\|\int_{t}^{t+h} \int_{0}^{s} C(s-r) \frac{1}{(r+h)^{\alpha}} d r \mathcal{A} v(t-s) d s\right\|_{H} \\
& +\left\|\int_{t}^{t+h} S(s) \mathcal{A} v_{0} \frac{1}{(t+h-s)^{\alpha}} d s\right\|_{H}=T_{1}+T_{2}+T_{3}+T_{4} .
\end{aligned}
$$

For $T_{1}$, we have that

$$
\begin{gathered}
T_{1} \leq \int_{0}^{t}\left\|\int_{0}^{s} C(s-r)\left(\frac{1}{(r+h)^{\alpha}}-\frac{1}{r^{\alpha}}\right) d r \mathcal{A} v(t-s)\right\|_{H} d s \\
\leq \int_{0}^{t} \int_{0}^{s}\left|\frac{1}{(r+h)^{\alpha}}-\frac{1}{r^{\alpha}}\right| d r\|\mathcal{A} v(t-s)\|_{H} d s \\
\leq \int_{0}^{t} \frac{(s+h)^{1-\alpha}-h^{1-\alpha}-s^{1-\alpha}}{1-\alpha} d s \sup _{t \in[0, T]}\|\mathcal{A} v(t)\|_{H} \rightarrow 0, \text { as }|h| \rightarrow 0 .
\end{gathered}
$$

For $T_{2}$, we have that

$$
\begin{aligned}
T_{2} & \leq \int_{0}^{t}\left\|S(s) \mathcal{A} v_{0}\left(\frac{1}{(t+h-s)^{\alpha}}-\frac{1}{(t-s)^{\alpha}}\right)\right\|_{H} d s \\
& \leq M_{S}\left\|\mathcal{A} v_{0}\right\|_{H} \int_{0}^{t}\left|\frac{1}{(t+h-s)^{\alpha}}-\frac{1}{(t-s)^{\alpha}}\right| d s
\end{aligned}
$$




$$
\leq M_{S}\left\|\mathcal{A} v_{0}\right\|_{H} \frac{(t+h)^{1-\alpha}-h^{1-\alpha}-t^{1-\alpha}}{1-\alpha} \rightarrow 0, \text { as }|h| \rightarrow 0
$$

For $T_{3}$, we have that

$$
\begin{gathered}
T_{3} \leq \int_{t}^{t+h}\left\|\int_{0}^{s} C(s-r) \frac{1}{(r+h)^{\alpha}} d r \mathcal{A} v(t-s)\right\|_{H} d s \\
\leq \int_{t}^{t+h} \int_{0}^{s} \frac{1}{(r+h)^{\alpha}} d r\|\mathcal{A} v(t-s)\|_{H} d s \\
\leq \int_{t}^{t+h} \frac{(s+h)^{1-\alpha}-h^{1-\alpha}}{1-\alpha} d s \sup _{t \in[0, T]}\|\mathcal{A} v(t)\|_{H} \rightarrow 0, \text { as }|h| \rightarrow 0 .
\end{gathered}
$$

For $T_{4}$, we have that

$$
\begin{gathered}
T_{4} \leq \int_{t}^{t+h}\left\|S(s) \mathcal{A} v_{0} \frac{1}{(t+h-s)^{\alpha}}\right\|_{H} d s \\
\leq M_{S}\left\|\mathcal{A} v_{0}\right\|_{H} \int_{t}^{t+h} \frac{1}{(t+h-s)^{\alpha}} d s \\
\leq M_{S}\left\|\mathcal{A} v_{0}\right\|_{H} \frac{h^{1-\alpha}}{1-\alpha} \rightarrow 0, \text { as }|h| \rightarrow 0 .
\end{gathered}
$$

Let $u_{1}, u_{2} \in B\left(v_{0}, R\right)$. Then, for $\left\|\Phi_{3}\left(u_{1}\right)-\Phi_{3}\left(u_{2}\right)\right\|_{\mathcal{C}}$ we find that:

$$
\left\|\Phi_{3}\left(u_{1}\right)-\Phi_{3}\left(u_{2}\right)\right\|_{\mathcal{C}}=\sup _{t \in[0, T]} \frac{c}{\rho A \Gamma(1-\alpha)}\left\|\int_{0}^{t} \int_{0}^{s} C(s-r)\left(u_{1}(r)-u_{2}(r)\right) d r \frac{1}{(t-s)^{\alpha}} d s\right\|_{H_{\mathcal{A}}} .
$$

Next, taking into account that

$$
\begin{gathered}
\left\|\int_{0}^{t} \int_{0}^{s} C(s-r) \mathcal{A}\left(u_{1}(r)-u_{2}(r)\right) d r \frac{1}{(t-s)^{\alpha}} d s\right\|_{H} \\
\leq \int_{0}^{t}\left\|\int_{0}^{s} C(s-r) \frac{1}{r^{\alpha}} d r \mathcal{A}\left(u_{1}(t-s)-u_{2}(t-s)\right)\right\|_{H} d s \\
\leq \int_{0}^{t} \frac{s^{1-\alpha}}{1-\alpha}\left\|\mathcal{A}\left(u_{1}(t-s)-u_{2}(t-s)\right)\right\|_{H} d s \\
\leq \int_{0}^{t} \frac{s^{1-\alpha}}{1-\alpha}\left\|\mathcal{A}\left(u_{1}(t-s)-u_{2}(t-s)\right)\right\|_{H} d s \\
\leq \frac{T^{2-\alpha}}{1-\alpha} \sup _{t \in[0, T]}\left\|\mathcal{A}\left(u_{1}(t)-u_{2}(t)\right)\right\|_{H}
\end{gathered}
$$

yields

$$
\left\|\Phi_{3}\left(u_{1}\right)-\Phi_{3}\left(u_{2}\right)\right\|_{\mathcal{C}} \leq \sqrt{1+M_{\mathcal{A}}^{2}} \frac{c T^{2-\alpha}}{(1-\alpha) \rho A \Gamma(1-\alpha)}\left\|u_{1}(t)-u_{2}(t)\right\|_{\mathcal{C}}
$$

Combining (32) of the proof of Theorem 1 and (58), we find that $\Phi: B\left(v_{0}, R\right) \rightarrow \mathcal{C}$ is a contraction, under the condition

$$
h=T \sqrt{1+M_{\mathcal{A}}^{2}}\left[\frac{c T^{1-\alpha}}{(1-\alpha) \rho A \Gamma(1-\alpha)}+\frac{3 A}{2 L I} M_{\mathcal{A}}^{2}\left(R+v_{0}^{\star}\right)^{2}\right]<1 .
$$


which is the expression (48) of Theorem 2. The conditions such that

$$
\left\|\Phi\left(v_{0}\right)-v_{0}\right\|_{\mathcal{C}}<R(1-h)
$$

are determined next. In this regard, observe that

$$
\begin{gathered}
\left\|\mathcal{A} \Phi\left(v_{0}\right)-\mathcal{A} v_{0}\right\|_{H} \leq \\
\left\|(C(t)-I) \mathcal{A} v_{0}\right\|_{H}+\left\|\mathcal{A} S(t) v_{1}\right\|_{H}+\left\|\frac{c}{\rho A \Gamma(1-\alpha)} \int_{0}^{t}\left(\int_{0}^{s} \mathcal{A} C(s-r) v_{0} d r-\mathcal{A} S(s) v_{0}\right) \frac{1}{(t-s)^{\alpha}} d s\right\|_{H} \\
+\frac{A}{2 L I}\left\|\int_{0}^{t} \mathcal{A} S(t-s)<\mathcal{A}^{1 / 2} v_{0}, v_{0}>_{H} \mathcal{A}^{1 / 2} v_{0} d s\right\|_{H}+\frac{1}{\rho A}\left\|\int_{0}^{t} \mathcal{A} S(t-s) q(s) d s\right\|_{H} \leq \\
2\left\|\mathcal{A} v_{0}\right\|_{H}+\left\|\mathcal{A}^{1 / 2} v_{1}\right\|_{H}+\frac{c}{\rho A \Gamma(1-\alpha)}\left(\int_{0}^{t}\left\|\int_{0}^{s} C(s-r) \frac{1}{r^{\alpha}} d r\right\| d s+\int_{0}^{t}\left\|S(t-s) \frac{1}{s^{\alpha}}\right\| d s\right)\left\|\mathcal{A} v_{0}\right\|_{H} \\
+\frac{T A}{2 L I}\left|<\mathcal{A}^{1 / 2} v_{0}, v_{0}>_{H}\right|\left\|\mathcal{A} v_{0}\right\|+\frac{T}{\rho A}\left\|\mathcal{A}^{1 / 2} q(s)\right\|_{H} \\
\leq 2 v_{0}^{\star}+v_{1}^{\star}+\frac{c T^{1-\alpha}\left(T+M_{S}\right)}{(1-\alpha) \rho A \Gamma(1-\alpha)} v_{0}^{\star}+\frac{T A}{2 L I} M_{\mathcal{A}}^{2}\left(v_{0}^{\star}\right)^{3}+\frac{T}{\rho A} q^{\star} .
\end{gathered}
$$

Thus,

$$
\left\|\Phi\left(v_{0}\right)-v_{0}\right\|_{\mathcal{C}}<\sqrt{1+M_{\mathcal{A}}^{2}}\left[\left(2+\frac{c T^{1-\alpha}\left(T+M_{S}\right)}{(1-\alpha) \rho A \Gamma(1-\alpha)}\right) v_{0}^{\star}+v_{1}^{\star}+\frac{T A}{2 L I} M_{\mathcal{A}}^{2}\left(v_{0}^{\star}\right)^{3}+\frac{T}{\rho A} q^{\star}\right]
$$

By considering expressions (59) and (60), the relation (49) of Theorem 2 is derived.

Returning to the IBVP (1), an implicit analytic expression for the solution $v(t, x)$, Eq. (3) is obtained. However, prior to presenting it, the following definitions are introduced:

$$
\begin{gathered}
v_{8}(t, x):=\frac{2 L}{\pi^{2}} \sqrt{\frac{\rho A}{E I}} \sum_{n=1}^{\infty}\left[\frac{1}{n^{2}} \sin \left(\sqrt{\frac{E I}{\rho A}} \frac{n^{2} \pi^{2}}{L^{2}} t\right)\left(\int_{0}^{L} v_{1}(z) \sin \left(\frac{n \pi}{L} z\right) d z\right) \sin \left(\frac{n \pi}{L} x\right)\right], \\
v_{9}(t, x):=\frac{2 c}{L \rho A \Gamma(1-\alpha)} \int_{0}^{t}\left\{\int_{0}^{s}\left\{\sum_{n=1}^{\infty}\left[\cos \left(\sqrt{\frac{E I}{\rho A}} \frac{n^{2} \pi^{2}}{L^{2}}(s-r)\right)\left(\int_{0}^{L} v(r, z) \sin \left(\frac{n \pi}{L} z\right) d z\right) \sin \left(\frac{n \pi}{L} x\right)\right]\right\} d r \frac{1}{(t-s)^{\alpha}}\right\} d s \\
v_{10}(t, x):=\frac{2 c L}{\pi^{2} \sqrt{E I \rho A} \Gamma(1-\alpha)} \int_{0}^{t}\left\{\sum_{n=1}^{\infty}\left[\frac{1}{n^{2}} \sin \left(\sqrt{\frac{E I}{\rho A}} \frac{n^{2} \pi^{2}}{L^{2}} s\right)\left(\int_{0}^{L} v_{0}(z) \sin \left(\frac{n \pi}{L} z\right) d z\right) \sin \left(\frac{n \pi}{L} x\right)\right] \frac{1}{(t-s)^{\alpha}}\right\} d s
\end{gathered}
$$

Thus, from Eqs. (35), (39) - (41) and (61) - (63), the implicit analytic solution $v(t, x)$ of the IBVP (1) is given by

$$
v(t, x)=v_{1}(t, x)+v_{8}(t, x)-v_{9}(t, x)+v_{10}(t, x)-v_{5}(t, x) \times v_{6}(t, x)+v_{7}(t, x) .
$$




\subsection{Purely elastic case}

For the elastic case $(\alpha=0)$, the term $v(t, x)-v(0, x)$ replaces the fractional derivative term, and thus, the equations admit the form

$$
\begin{aligned}
& \rho A \frac{\partial^{2} v}{\partial t^{2}}(t, x)+c(v(t, x)-v(0, x))+E I \frac{\partial^{4} v}{\partial x^{4}}(t, x)-N \frac{\partial^{2} v}{\partial x^{2}}(t, x)=q(t, x), \text { a.e. on }(0, T) \times(0, L), \\
& v(0, x)=v_{0}(x), \frac{\partial v}{\partial t}(0, x) \quad=v_{1}(x), \text { a.e. on }(0, L), \\
& v(t, 0)=v(t, L)=\frac{\partial^{2} v}{\partial x^{2}}(t, 0)=\frac{\partial^{2} v}{\partial x^{2}}(t, L) \quad=0, \text { a.e. on }(0, T) .
\end{aligned}
$$

The IBVP (65) derives as follows:

$$
\left.\begin{array}{c}
v^{\prime \prime}(t)+\mathcal{A} v(t)+\frac{c}{\rho A}\left(v(t)-v_{0}\right)+\frac{A}{2 L I}<\mathcal{A}^{1 / 2} v(t), v(t)>_{H} \mathcal{A}^{1 / 2} v(t)=\frac{1}{\rho A} q(t), \text { a.e. on }(0, T), \\
v(0)=v_{0}, v^{\prime}(0)=v_{1},
\end{array}\right\}
$$

Definition 3. A twice differentiable function $v:[0, T] \rightarrow H$, is called a solution of the IVP (66) if

(i) $v(t) \in D(\mathcal{A}), \quad t \in[0, T]$,

(ii) $v(t)=C(t) v_{0}+S(t) v_{1}-\frac{c}{\rho A} \int_{0}^{t} S(t-s)\left(v(s)-v_{0}\right) d s-$

$$
\frac{A}{2 L I} \int_{0}^{t} S(t-s)<\mathcal{A}^{1 / 2} v(s), v(s)>_{H} \mathcal{A}^{1 / 2} v(s) d s+\frac{1}{\rho A} \int_{0}^{t} S(t-s) q(s) d s, t \in[0, T] .
$$

Applying the same techniques as in the proofs of Theorem 1 and Theorem 2, it is possible to find similar relations to (28), (29) and (48), (49) for the existence of a unique fixed point in $B\left(v_{0}, R\right)$ for the IVP (66). This unique fixed point has the form of Definition 3. However, note that a more convenient form of the solution of the IVP (66) than this of Definition 3 can be found. To this aim, for $c>0$, the operator $\mathcal{A}_{1}: D\left(\mathcal{A}_{1}\right) \rightarrow H$ is defined as

$$
\mathcal{A}_{1} v(t, \cdot)=\left(\mathcal{A}+\frac{c}{\rho A} \mathcal{I}\right) v(t, \cdot)=\left(\frac{E I}{\rho A} \frac{\partial^{4} v}{\partial x^{4}}+\frac{c}{\rho A} \mathcal{I}\right)(t, \cdot)
$$

with

$$
D\left(\mathcal{A}_{1}\right)=D(\mathcal{A})
$$

The operator $\mathcal{A}_{1}=\mathcal{A}+\frac{c}{\rho A} \mathcal{I}$ is also positive and self-adjoint on $H$ with eigenvalues

$$
\mu_{n}=\frac{E I}{\rho A}\left(\frac{n \pi}{L}\right)^{4}+\frac{c}{\rho A}, n \in \mathbb{N}
$$


and corresponding eigenvectors

$$
e_{n}(x)=\sqrt{\frac{2}{L}} \sin \left(\frac{n \pi}{L} x\right), n \in \mathbb{N}, x \in(0, L) .
$$

The corresponding $C_{1}(t)$ and $S_{1}(t)$ on $H$, generated by $-\mathcal{A}_{1}$, take the form

$$
C_{1}(t) y=\sum_{n=1}^{\infty} \cos \left(\sqrt{\frac{E I}{\rho A} \frac{n^{4} \pi^{4}}{L^{4}}+\frac{c}{\rho A}} t\right)<y, e_{n}>_{H} e_{n} \text {, for } y \in H .
$$

and

$$
S_{1}(t) y=\sum_{n=1}^{\infty} \sqrt{\frac{\rho A}{E I \frac{n^{4} \pi^{4}}{L^{4}}+c}} \sin \left(\sqrt{\frac{E I}{\rho A} \frac{n^{4} \pi^{4}}{L^{4}}+\frac{c}{\rho A}} t\right)<y, e_{n}>_{H} e_{n} \text {, for } y \in H .
$$

The IBVP (65) takes now the following form of a second order nonlinear ODE in $H$ :

$$
\begin{gathered}
v^{\prime \prime}(t)+\mathcal{A}_{1} v(t)+\frac{A}{2 L I}<\mathcal{A}^{1 / 2} v(t), v(t)>_{H} \mathcal{A}^{1 / 2} v(t)=\frac{c}{\rho A} v_{0}+\frac{1}{\rho A} q(t), \text { a.e. on }(0, T), \\
v(0)=v_{0}, v^{\prime}(0)=v_{1},
\end{gathered}
$$

Definition 4. A twice differentiable function $v:[0, T] \rightarrow H$, is called a solution of the IVP (68) if

(i) $v(t) \in D(\mathcal{A}), \quad t \in[0, T]$,

(ii) $v(t)=C_{1}(t) v_{0}+S_{1}(t) v_{1}-\frac{A}{2 L I} \int_{0}^{t} S_{1}(t-s)<\mathcal{A}^{1 / 2} v(s), v(s)>_{H} \mathcal{A}^{1 / 2} v(s) d s+$

$$
\frac{c}{\rho A} \int_{0}^{t} S_{1}(t-s) v_{0} d s+\frac{1}{\rho A} \int_{0}^{t} S_{1}(t-s) q(s) d s, t \in[0, T] .
$$

Once again, applying the same techniques as in the proofs of Theorems 1 and 2, we are able to find similar relations to (28), (29) and (48), (49) for the existence of a unique fixed point in $B\left(v_{0}, R\right)$ for the IVP (68) which has the form of Definition 4.

Returning to the IBVP (65), an implicit analytic expression for the solution $v(t, x)$, Eq. (3) is obtained. In this regard, considering the definition

$v_{11}(t, x):=\frac{2 c L}{\pi^{2} \sqrt{E I \rho A}} \int_{0}^{t}\left\{\sum_{n=1}^{\infty}\left[\frac{1}{n^{2}} \sin \left(\sqrt{\frac{E I}{\rho A}} \frac{n^{2} \pi^{2}}{L^{2}}(t-s)\right)\left(\int_{0}^{L}\left(v(s, z)-v_{0}(z)\right) \sin \left(\frac{n \pi}{L} z\right) d z\right) \sin \left(\frac{n \pi}{L} x\right)\right]\right\} d s$

and employing Eqs. (35), (39) - (41), (61) and (70), the implicit analytic solution $v(t, x)$ of the IBVP (65) is given by

$$
v(t, x)=v_{1}(t, x)+v_{8}(t, x)-v_{11}(t, x)-v_{5}(t, x) \times v_{6}(t, x)+v_{7}(t, x) .
$$

Finally, to obtain a more convenient form of the solution of the IBVP (65), corresponding to the formulation of the IVP (68), the following result is proved first: 
Lemma 5. Let $v(s) \in D\left(\mathcal{A}^{1 / 2}\right), s \in[0, T], \mathcal{P}$-a.s. Then, the non-linear term in the solution map of Definition 4 , considered as a function from $[0, T] \times[0, L]$ to $\mathbb{R}$, takes the expression

$$
\begin{gathered}
\frac{A}{2 L I} \int_{0}^{t} S_{1}(t-s)<\mathcal{A}^{1 / 2} v(s), v(s)>_{H} \mathcal{A}^{1 / 2} v(s) d s= \\
\frac{4 \pi^{4}}{L^{7}} \sqrt{\frac{E A}{\rho I}} \int_{0}^{t}\left\{\sum_{n=1}^{\infty}\left[n^{2}\left(\int_{0}^{L} v(s, z) \sin \left(\frac{n \pi}{L} z\right) d z\right)^{2}\right] \times\right. \\
\left.\sum_{n=1}^{\infty}\left[n^{2} \sqrt{\frac{E I}{E I \frac{n^{4} \pi^{4}}{L^{4}}+c}} \sin \left(\sqrt{\frac{E I}{\rho A} \frac{n^{4} \pi^{4}}{L^{4}}+\frac{c}{\rho A}}(t-s)\right)\left(\int_{0}^{L} v(s, z) \sin \left(\frac{n \pi}{L} z\right) d z\right) \sin \left(\frac{n \pi}{L} x\right)\right]\right\} d s .
\end{gathered}
$$

Proof. By similar computations as in the proof of Lemma 1 in [25], we obtain that

$$
\begin{aligned}
& S_{1}(t-s) \mathcal{A}^{1 / 2} v(s)=\sum_{n=1}^{\infty}\left[\sqrt{\frac{\rho A}{E I \frac{n^{4} \pi^{4}}{L^{4}}+c}} \sin \left(\sqrt{\frac{E I}{\rho A} \frac{n^{4} \pi^{4}}{L^{4}}+\frac{c}{\rho A}}(t-s)\right)<\mathcal{A}^{1 / 2} v(s), e_{n}>_{H} e_{n}\right]= \\
& \sum_{n=1}^{\infty}\left[\sqrt{\frac{\rho A}{E I \frac{n^{4} \pi^{4}}{L^{4}}+c}} \sin \left(\sqrt{\frac{E I}{\rho A} \frac{n^{4} \pi^{4}}{L^{4}}+\frac{c}{\rho A}}(t-s)\right)\left\langle\left[\sum_{m=1}^{\infty} \sqrt{\frac{E I}{\rho A}} \frac{m^{2} \pi^{2}}{L^{2}}<v(s), e_{m}>_{H} e_{m}\right], e_{n}\right\rangle_{H} e_{n}\right]= \\
& \sum_{n=1}^{\infty}\left[\frac{n^{2} \pi^{2}}{L^{2}} \sqrt{\frac{E I}{E I \frac{n^{4} \pi^{4}}{L^{4}}+c}} \sin \left(\sqrt{\frac{E I}{\rho A} \frac{n^{4} \pi^{4}}{L^{4}}+\frac{c}{\rho A}}(t-s)\right)<v(s), e_{n}>_{H} e_{n}\right]= \\
& \frac{2 \pi^{2}}{L^{3}} \sum_{n=1}^{\infty}\left[n^{2} \sqrt{\frac{E I}{E I \frac{n^{4} \pi^{4}}{L^{4}}+c}} \sin \left(\sqrt{\frac{E I}{\rho A} \frac{n^{4} \pi^{4}}{L^{4}}+\frac{c}{\rho A}}(t-s)\right)\left(\int_{0}^{L} v(s, z) \sin \left(\frac{n \pi}{L} z\right) d z\right) \sin \left(\frac{n \pi}{L} x\right)\right], \quad s, t \in[0, T],
\end{aligned}
$$

Furthermore, by Lemma 3 and the spectral representation of $\mathcal{A}^{1 / 2}$, we have that

$$
<\mathcal{A}^{1 / 2} v(s), v(s)>_{H}=\frac{2 \pi^{2}}{L^{3}} \sqrt{\frac{E I}{\rho A}} \sum_{n=1}^{\infty}\left[n^{2}\left(\int_{0}^{L} v(s, z) \sin \left(\frac{n \pi}{L} z\right) d z\right)^{2}\right]
$$

Applying Lemma 5 to the solution map of Definition 4, the solution of the IBVP (65), corresponding to the form of the IVP (68), is presented in the following. In this regard, defining

$$
\begin{aligned}
& \tilde{v}_{1}(t, x):=\frac{2}{L} \sum_{n=1}^{\infty}\left[\cos \left(\sqrt{\frac{E I}{\rho A} \frac{n^{4} \pi^{4}}{L^{4}}+\frac{c}{\rho A}} t\right)\left(\int_{0}^{L} v_{0}(z) \sin \left(\frac{n \pi}{L} z\right) d z\right) \sin \left(\frac{n \pi}{L} x\right)\right], \\
& \left.\tilde{v}_{6}(t, x):=\sum_{n=1}^{\infty}\left[n^{2} \sqrt{\frac{E I}{E I \frac{n^{4} \pi^{4}}{L^{4}}+c}} \sin \left(\sqrt{\frac{E I}{\rho A} \frac{n^{4} \pi^{4}}{L^{4}}+\frac{c}{\rho A}}(t-s)\right)\left(\int_{0}^{L} v(s, z) \sin \left(\frac{n \pi}{L} z\right) d z\right) \sin \left(\frac{n \pi}{L} x\right)\right]\right\} d s \\
& \tilde{v}_{7}(t, x):=\frac{2}{L \sqrt{\rho A}} \int_{0}^{t}\left\{\sum_{n=1}^{\infty}\left[\frac{1}{\sqrt{E I \frac{n^{4} \pi^{4}}{L^{4}}+c}} \sin \left(\sqrt{\frac{E I}{\rho A} \frac{n^{4} \pi^{4}}{L^{4}}+\frac{c}{\rho A}}(t-s)\right)\left(\int_{0}^{L} q(s, z) \sin \left(\frac{n \pi}{L} z\right) d z\right) \sin \left(\frac{n \pi}{L} x\right)\right]\right\} d s
\end{aligned}
$$




$$
\begin{aligned}
& \tilde{v}_{8}(t, x):=\frac{2 \sqrt{\rho A}}{L} \sum_{n=1}^{\infty}\left[\frac { 1 } { \sqrt { E I \frac { n ^ { 4 } \pi ^ { 4 } } { L ^ { 4 } } + c } } \operatorname { s i n } \left(\sqrt{\left.\left.\frac{E I}{\rho A} \frac{n^{4} \pi^{4}}{L^{4}}+\frac{c}{\rho A} t\right)\left(\int_{0}^{L} v_{1}(z) \sin \left(\frac{n \pi}{L} z\right) d z\right) \sin \left(\frac{n \pi}{L} x\right)\right]}\right.\right. \\
& \tilde{v}_{10}(t, x):=\frac{2 c}{L \sqrt{\rho A}} \int_{0}^{t}\left\{\sum_{n=1}^{\infty}\left[\frac{1}{\sqrt{E I \frac{n^{4} \pi^{4}}{L^{4}}+c}} \sin \left(\sqrt{\frac{E I}{\rho A} \frac{n^{4} \pi^{4}}{L^{4}}+\frac{c}{\rho A}}(t-s)\right)\left(\int_{0}^{L} v_{0}(z) \sin \left(\frac{n \pi}{L} z\right) d z\right) \sin \left(\frac{n \pi}{L} x\right)\right]\right\} d s
\end{aligned}
$$

and considering Eqs. (39) and (73) - (76), the implicit analytic solution $v(t, x)$ of the IBVP (65) is given by

$$
v(t, x)=\tilde{v}_{1}(t, x)+\tilde{v}_{8}(t, x)-\frac{2 \pi^{2}}{L} v_{5}(t, x) \times \tilde{v}_{6}(t, x)+\tilde{v}_{10}(t, x)+\tilde{v}_{7}(t, x) .
$$

\section{Time regularity of the solutions}

For all the solution forms in the viscoelastic, and the purely viscous and the purely elastic cases, the following theorem is obtained. Note that to avoid repetition and for the sake of concise presentation, the corresponding proof is omitted taking into account that similar techniques are utilized as the ones employed in the proofs of Theorems 1 and 2 .

Theorem 3. Suppose that assumptions, $(26)$ and $(27)$ hold, and $v \in \mathcal{C}$. Then $v^{\prime} \in \mathcal{C}\left(0, T ; H_{\mathcal{A}^{1 / 2}}\right)$ and $v^{\prime \prime} \in \mathcal{C}(0, T ; H)$.

In what follows, we present the first and second derivatives of the solutions for each case.

\subsection{Viscous case}

Differentiating formally the solution map of Definition 1 yields

$$
\begin{aligned}
v^{\prime}(t)=\left(-\mathcal{A} S(t)+\frac{c}{\rho A} C(t)\right) v_{0}+C(t) v_{1}+\frac{c}{\rho A} \int_{0}^{t} \mathcal{A} S(t-s) v(s) d s-\frac{c}{\rho A} v(t) \\
-\frac{A}{2 L I} \int_{0}^{t} C(t-s)<\mathcal{A}^{1 / 2} v(s), v(s)>_{H} \mathcal{A}^{1 / 2} v(s) d s+\frac{1}{\rho A} \int_{0}^{t} C(t-s) q(s) d s, t \in[0, T] .
\end{aligned}
$$

Since assumptions, $(26)$ and $(27)$, hold, and $v \in \mathcal{C}\left(0, T ; H_{\mathcal{A}}\right)$, applying similar techniques to the proof of Theorem 1, it can be proved that $v^{\prime}(t) \in D\left(\mathcal{A}^{1 / 2}\right), t \in[0, T]$, and that $v^{\prime}$ is continuous under the usual norm of $\mathcal{C}\left(0, T ; H_{\mathcal{A}^{1 / 2}}\right)$. Furthermore, differentiating the above expression, we derive that 


$$
\begin{array}{r}
v^{\prime \prime}(t)=\left(-\mathcal{A} C(t)-\frac{c}{\rho A} \mathcal{A} S(t)\right) v_{0}-\mathcal{A} S(t) v_{1}+\frac{c}{\rho A} \int_{0}^{t} \mathcal{A} C(t-s) v(s) d s-\frac{c}{\rho A} v^{\prime}(t) \\
+\frac{A}{2 L I} \int_{0}^{t} \mathcal{A} S(t-s)<\mathcal{A}^{1 / 2} v(s), v(s)>_{H} \mathcal{A}^{1 / 2} v(s) d s-\frac{A}{2 L I}<\mathcal{A}^{1 / 2} v(t), v(t)>_{H} \mathcal{A}^{1 / 2} v(t) \\
-\frac{1}{\rho A} \int_{0}^{t} \mathcal{A} S(t-s) q(s) d s+\frac{1}{\rho A} q(t), t \in[0, T] .
\end{array}
$$

By similar techniques applied in the proof of Theorem 1, in conjunction with the continuity of the inner product and $v^{\prime}$, it can be proved that $v^{\prime \prime}(t)$ is a well defined $H$-valued function for $t \in[0, T]$, and that $v^{\prime \prime}$ is continuous under the usual norm of $\mathcal{C}(0, T ; H)$.

\subsection{Viscoelastic case}

Applying Lemma 4 (Result 2) to differentiate the solution map of Definition 2 leads to

$$
\begin{array}{r}
v^{\prime}(t)=-\mathcal{A} S(t) v_{0}+C(t) v_{1}+\frac{c}{\rho A \Gamma(1-\alpha)} \int_{0}^{t}\left(\int_{0}^{s} \mathcal{A} S(s-r) v(r) d r-v(s)+C(s) v_{0}\right) \frac{1}{(t-s)^{\alpha}} d s \\
-\frac{A}{2 L I} \int_{0}^{t} C(t-s)<\mathcal{A}^{1 / 2} v(s), v(s)>_{H} \mathcal{A}^{1 / 2} v(s) d s+\frac{1}{\rho A} \int_{0}^{t} C(t-s) q(s) d s, t \in[0, T] .
\end{array}
$$

Since assumptions, (26) and (27), hold, and $v \in \mathcal{C}\left(0, T ; H_{\mathcal{A}}\right)$, applying similar techniques to the ones for proving Theorems 1 and 2 , it can shown that $v^{\prime}(t) \in D\left(\mathcal{A}^{1 / 2}\right), t \in[0, T]$, and that $v^{\prime}$ is continuous under the usual norm of $\mathcal{C}\left(0, T ; H_{\mathcal{A}^{1 / 2}}\right)$. Furthermore, differentiating the above expression and applying Lemma 4 (Result 3), we obtain that

$$
\begin{array}{r}
v^{\prime \prime}(t)=-\mathcal{A} C(t) v_{0}-\mathcal{A} S(t) v_{1}+\frac{c}{\rho A \Gamma(1-\alpha)} \int_{0}^{t}\left(v^{\prime}(s)-\int_{0}^{s} \mathcal{A} S(s-r) v^{\prime}(r) d r\right) \frac{1}{(t-s)^{\alpha}} d s \\
+\frac{A}{2 L I} \int_{0}^{t} \mathcal{A} S(t-s)<\mathcal{A}^{1 / 2} v(s), v(s)>_{H} \mathcal{A}^{1 / 2} v(s) d s-\frac{A}{2 L I}<\mathcal{A}^{1 / 2} v(t), v(t)>_{H} \mathcal{A}^{1 / 2} v(t) \\
-\frac{1}{\rho A} \int_{0}^{t} \mathcal{A} S(t-s) q(s) d s+\frac{1}{\rho A} q(t), t \in[0, T] .
\end{array}
$$

By resorting to similar techniques as the ones involved in the proofs of Theorems 1 and 2, in conjunction with the continuity of the inner product and $v^{\prime}$, it can be proved that $v^{\prime \prime}(t)$ is a well defined $H$-valued function for $t \in[0, T]$, and that $v^{\prime \prime}$ is continuous under the usual norm of $\mathcal{C}(0, T ; H)$. 


\subsection{Elastic case}

Differentiating formally the solution map of Definition 3 yields

$$
\begin{aligned}
v^{\prime}(t)=-\mathcal{A} S(t) v_{0}+C(t) v_{1}-\frac{c}{\rho A} \int_{0}^{t} C(t-s)\left(v(s)-v_{0}\right) d s \\
\quad-\frac{A}{2 L I} \int_{0}^{t} C(t-s)<\mathcal{A}^{1 / 2} v(s), v(s)>_{H} \mathcal{A}^{1 / 2} v(s) d s+\frac{1}{\rho A} \int_{0}^{t} C(t-s) q(s) d s, t \in[0, T] .
\end{aligned}
$$

Since assumptions, (26) and (27), hold, and $v \in \mathcal{C}\left(0, T ; H_{\mathcal{A}}\right)$, applying similar techniques to the ones in the proof Theorem 1 , it can shown that $v^{\prime}(t) \in D\left(\mathcal{A}^{1 / 2}\right), t \in[0, T]$, and that $v^{\prime}$ is continuous under the usual norm of $\mathcal{C}\left(0, T ; H_{\mathcal{A}^{1 / 2}}\right)$. Furthermore, differentiating the above expression leads to

$$
\begin{array}{r}
v^{\prime \prime}(t)=-\mathcal{A} C(t) v_{0}-\mathcal{A} S(t) v_{1}+\int_{0}^{t} \mathcal{A} S(t-s)\left(v(s)-v_{0}\right) d s-\frac{c}{\rho A}\left(v(t)-v_{0}\right) \\
+\frac{A}{2 L I} \int_{0}^{t} \mathcal{A} S(t-s)<\mathcal{A}^{1 / 2} v(s), v(s)>_{H} \mathcal{A}^{1 / 2} v(s) d s-\frac{A}{2 L I}<\mathcal{A}^{1 / 2} v(t), v(t)>_{H} \mathcal{A}^{1 / 2} v(t) \\
-\frac{1}{\rho A} \int_{0}^{t} \mathcal{A} S(t-s) q(s) d s+\frac{1}{\rho A} q(t), t \in[0, T] .
\end{array}
$$

Clearly, $v^{\prime \prime}(t)$ is a well defined $H$-valued function for $t \in[0, T]$, and is continuous under the usual norm of $\mathcal{C}(0, T ; H)$. Hence, for all the three cases, the solution map $v:[0, T] \rightarrow H$, is twice continuously differentiable.

\section{Concluding remarks}

In this study, implicit analytic solutions have been obtained for a nonlinear fractional PDE modeling the dynamics of a deterministically excited viscoelastic nonlinear Euler-Bernoulli beam. This has been yielded, first, by recasting the nonlinear PDE as a second order nonlinear IVP in the Hilbert space $L^{2}$. Next, the abstract theory of second order ODEs in Hilbert spaces, which involves special families of operators, has been employed for deriving a variation of parameters representation for the solution map of this problem. In this regard, local existence and uniqueness of a fixed point for this solution map have been obtained as well. Next, representing both the nonlinear and the fractional terms in suitable forms and invoking properties of the fractional derivative as well as properties of the cosine and sine families of operators, this implicit solution has been expressed in an analytic derivative-free 
form. Note that the solutions for the limiting cases $a=0$ (elastic case) and $a=1$ (viscous case) have also been derived in similar forms.

The herein derived results are of considerable importance given the increasing number of application of fractional calculus in diverse areas of engineering and science [37]. Apart from the theoretical merit of the determined implicit form solutions, these closed-form expressions can be used, potentially, as an important step to derive very efficient and fast numerical solutions. The latter is identified as a topic for a following paper.

\section{Acknowledgements}

We thank Mansoor Saburov for useful discussions on the generalizations of the Banach Contraction Principle.

\section{References}

[1] O. P. Agrawal, Stochastic analysis of dynamic systems containing fractional derivatives. J. Sound Vib. 247 (5) (2001) 927-938.

[2] G. Alotta, M. Di Paola, G. Failla, F. P. Pinnola. On the dynamics of non-local fractional viscoelastic beams under stochastic agencies. Compos. Part B-Eng., 137 (2018) 102-110.

[3] K. Balachandran, J. Y. Park, Existence of solutions of a class of abstract second order nonlinear integrodifferential equations, J. Appl. Math. Stoch. Anal. 15 (2) (2002) 115-124.

[4] P. Balasubramaniam, J.Y. Park, Nonlocal Cauchy problem for second order stochastic evolution equations in Hilbert spaces, Dynamic Syst. Appl., 16 (2007) 713-728.

[5] M. Caputo, Linear models of dissipation whose Q is almost frequency independent-II, Geophys. J. Int. 13 (5) (1967) 529-539.

[6] M. Caputo, Elasticità e dissipazione, Zanichelli, Bologna, Italy, 1969.

[7] N. Challamel, I. Elishakoff, A brief history of first-order shear-deformable beam and plate models, Mechanics Res. Commun. (2019) (in press).

[8] N. Colinas-Armijo, S. Cutrona, M. Di Paola, A. Pirrotta, Fractional viscoelastic beam under torsion, Commun. Nonlinear Sci, 48 (2017), 278-287 
[9] G. Di Blasio, K. Kunisch, E. Sinestrari, Mathematical models for the elastic beam with structural damping, Appl. Anal., 48 (1-4) (1993) 133-156.

[10] S. Di Lorenzo, M. Di Paola, F. P. Pinnola, A. Pirrotta, Stochastic response of fractionally damped beams, Probabilistic Eng. Mech. 35 (2014) 37-43.

[11] A. Di Matteo, I. A. Kougioumtzoglou, A. Pirrotta, P. D. Spanos, M. Di Paola, Stochastic response determination of nonlinear oscillators with fractional derivatives elements via the Wiener path integral, Probabilistic Eng. Mech. 38 (2014) 127-135.

[12] A. Di Matteo, F. Lo Iacono, G. Navarra, A. Pirrotta, Innovative modeling of tuned liquid column damper motion. Commun. Nonlinear Sci, 23(1-3) (2015) 229-244.

[13] M. Di Paola, R. Heuer, A. Pirrotta, Fractional visco-elastic Euler-Bernoulli beam, Int. J. Solids Struct., 50(22-23) (2013), 3505-3510

[14] K. J. Engel, R. Nagel, One-Parameter Semigroups for Linear Evolution Equation, Springer Verlag, New York, 2000.

[15] H. O. Fattorini, Ordinary differential equations in linear topological spaces, I, J. Differ. Equations 5 (1968) $72-105$.

[16] H. O. Fattorini, Ordinary differential equations in linear topological spaces, II, J. Differ. Equations 6 (1969) 50-70.

[17] W. E. Fitzgibbon, Global existence and boundedness of solutions to the extensible beam equation, SIAM J. Math. Anal. 13 (1982) 739-745.

[18] V.C. Fragkoulis, I.A. Kougioumtzoglou, A. A. Pantelous, M. Beer, Non-stationary response statistics of nonlinear oscillators with fractional derivative elements under evolutionary stochastic excitation, Nonlinear Dynam., 97(4) (2019), 2291-2303.

[19] A. Gemant, A method of analyzing experimental results obtained from elasto-viscous bodies, Physics 7 (8) (1936) 311-317.

[20] D. Y. Gao, J. Machalová, H. Netuka, Mixed finite element solutions to contact problems of nonlinear Gao beam on elastic foundation. Nonlinear Anal.-Real., 22, (2015) 537-550.

[21] I. A. Kougioumtzoglou, P. D. Spanos, Harmonic wavelets based response evolutionary power spectrum determination of linear and nonlinear oscillators with fractional derivative elements, Int. J. Nonlinear Mech. 80 (2016) 66-75. 
[22] A. Latif, Banach Contraction Principle and Its Generalizations. In: Almezel S, Ansari Q, Khamsi M, editors. Topics in Fixed Point Theory. Springer, Cham 2014 33-64.

[23] K. B. Liaskos, A. A. Pantelous, I. G. Stratis. Linear stochastic degenerate Sobolev equations and applications. Int. J. Control, 88(12) (2015) 2538-2553.

[24] K. B. Liaskos, I. G. Stratis., A. A. Pantelous. Stochastic degenerate Sobolev equations: well posedness and exact controllability. Math. Method. Appl. Sci, 41(3) (2018) 1025-1032.

[25] K. B. Liaskos, A. A. Pantelous, I. A. Kougioumtzoglou, A. T. Meimaris, Implicit analytic solutions for the linear stochastic partial differential beam equation with fractional derivative terms, Syst. Control Lett. 121 (2018) 38-49.

[26] F. Mainardi, Fractional calculus: some basic problems in continuum and statistical mechanics, In: Carpinter A, Mainardi F, editors. Fractals and fractional calculus in continuum mechanics. New York: Springer-Verlag, (1997) 291-348.

[27] N. I. Mahmudov, M. A. McKibben, Abstract second-order damped McKean-Vlasov stochastic evolution equations, Stoch. Anal. Appl. 24 (2) (2006) 303-328.

[28] M. A. McKibben, Second-order damped functional stochastic evolution equations in Hilbert space, Dyn. Syst. Appl. 12, 467 (2003).

[29] P. G. Nutting, A new general law of deformation. J. Franklin I., 191 (5) (1921) 679-685.

[30] A. Pazy, Semigroups of Linear Operators and Applications to Partial Differential Equations, Springer Verlag, New York, 1983.

[31] A. Pirrotta, S. Cutrona, S. Di Lorenzo, Fractional visco-elastic Timoshenko beam from elastic Euler-Bernoulli beam, Acta Mechanica, 226(1) (2015) 179-189.

[32] A. Pirrotta, S. Cutrona, S. Di Lorenzo, A. D. Matteo Fractional visco-elastic Timoshenko beam deflection via single equation. Int.J. Numer. Meth. Eng., 104(9) (2015) 869-886.

[33] S.S. Rao, Mechanical Vibrations, Addison Wesley, 1995.

[34] Y. A. Rossikhin, M. V. Shitikova, Application of fractional calculus for dynamic problems of solid mechanics: Novel trends and recent results. Appl. Mech. Reviews 63 (1) (2009) 010801.

[35] S. Sarkar, J. N. Reddy, Exploring the source of non-locality in the Euler-Bernoulli and Timoshenko beam models, Int. J. Eng. Sci., 104 (2016), 110-115

[36] P. D. Spanos, G. Malara, Nonlinear Random Vibrations of Beams with Fractional Derivative Elements, ASCE J. Eng. Mech. 140 (9) (2014) 04014069. 
[37] H. Sun, Y. Zhang, D. Baleanu, W. Chen, Y. Chen. A new collection of real world applications of fractional calculus in science and engineering. Commun. Nonlinear Sci., 64 (2018) 213-231.

[38] C. C. Travis, G. F. Webb, Cosine families and abstract nonlinear second order differential equations, Acta Math. Acad. Sci. Hung. 32 (1978) 75-96.

[39] C. C. Travis, G. F. Webb, Second order differential equations in Banach space. In: Proceedings International Symposium on Nonlinear Equations in Abstract Spaces, pp. 331-361. Academic Press, New York, 1987. 